\title{
Arnold Krawietz \\ Surface tension and reaction stresses of a linear incompressible second gradient fluid
}

Received: 29 September 2020 / Accepted: 2 November 2020 / Published online: 10 January 2021

(C) The Author(s) 2021

\begin{abstract}
The paper starts with a detailed investigation of the boundary conditions at free and fixed boundaries of any second gradient material and clarifies whether a surface tension is to be expected. The classical approach to the reaction stresses of higher gradient materials leaves a vast indeterminacy in most boundary value problems. An advanced approach is presented that yields much more definite distributions of the reaction stresses and consequently also of the surface tension. It starts from a compressible fluid the stiffness and bulk viscosity of which tend to infinity. Furthermore, the complete set of restrictions on the material parameters of linear incompressible second gradient fluids is derived from the postulate of nonnegative dissipation. Finally, a stirring process with a free surface is studied as an example. The results are based on the numerical solution of the boundary value problems of three partial differential equations of second, fourth and sixth order, respectively.
\end{abstract}

Keywords Second gradient fluid - Incompressible $\cdot$ Surface tension $\cdot$ Reaction stresses $\cdot$ Restrictions on material parameters $\cdot$ Stirring

\section{Introduction}

The constitutive model of a linear second gradient fluid is a natural extension of the classical Navier-Stokes fluid. Incompressibility is an assumption of practical importance. It was St.-Venant [1] who conjectured in 1869 that the consideration of higher velocity gradients might improve the description of turbulent flows. But this approach was not elaborated for a long time. Trostel [2] and his coworkers in Berlin took care of the subject since 1985. There are also plenty of publications on capillary fluids (e.g., [3-6]) which are extensions of Euler's inviscid compressible fluid.

The paper at hand reveals several aspects of second gradient materials in general and especially second gradient linear fluids that seem not to have been in the focus of former investigations, e.g., $[3,7,8]$.

Section 2 derives boundary conditions from the principle of virtual velocities. It turns out that a free surface of a second gradient material - whatever be its constitutive behavior - is endowed with a crust shell that is the basis of a surface tension of dimension force per length.

Section 3 elaborates the properties of a compressible linear second gradient fluid, while Sect. 4 carries out the reduction to the incompressible case. There is only a pressure field $p$ as a reaction quantity with the Navier-Stokes fluid. It can be computed together with the velocity field if there is a free surface. In contrast to this, a vector field $\mathbf{q}$ appears as an additional reaction quantity with a second gradient material. This is not uniquely determined even in the presence of a free surface. This unsatisfactory situation is defused by an

Communicated by Andreas Öchsner.

A. Krawietz $(\varangle)$

Berlin, Germany

E-mail: krawietz@t-online.de 
advanced approach: The incompressible material is regarded as the limiting case of a compressible one the stiffness and bulk viscosity of which tend to infinity.

Section 5 presents restrictions on the material parameters.

Sections 6, 7 and 8 examine a stirring process as an example. It turns out that not only the primary flow but also a secondary flow must be taken into account in order to determine the shape of the free surface. The reaction fields $p$ and $\mathbf{q}$ remain vague unless the advanced approach is applied.

The results can be visualized if special assumptions on the geometry and the material parameters are made. This is done in Sect. 9. The boundary value problems of three partial differential equations of second, fourth and sixth order, respectively, are attacked by means of a difference scheme, and some results are discussed and visualized.

\section{Internal and external virtual power}

The properties of second gradient materials are extensively elaborated in a compendium by Bertram [9]. An article of Bertram is especially devoted to viscous gradient fluids [10]. Details can also be found in a recent book [11].

The virtual stress power per unit volume of a second gradient material is a linear form of the first and second virtual velocity gradients $\delta \mathbf{v} \otimes \nabla$ and $\delta \mathbf{v} \otimes \nabla \otimes \nabla$, respectively:

$$
\delta \pi_{i}=\mathbf{T}_{2}: \delta \mathbf{v} \otimes \nabla+\mathbf{T}_{3}: \cdot \delta \mathbf{v} \otimes \nabla \otimes \nabla
$$

(Notation: A dot denotes a contraction. The multiple contraction is written $\mathbf{a} \otimes \mathbf{b} \otimes \mathbf{c}: \cdot \mathbf{d} \otimes \mathbf{e} \otimes \mathbf{f}=\mathbf{a} \cdot \mathbf{d} \mathbf{b} \cdot \mathbf{e} \mathbf{c} \cdot \mathbf{f}$ and $\mathbf{a} \otimes \mathbf{b}: \mathbf{d} \otimes \mathbf{e}=\mathbf{a} \cdot \mathbf{d} \mathbf{b} \cdot \mathbf{e}$.) The second-order stress tensor $\mathbf{T}_{2}$ is symmetric due to the postulate of invariance of the virtual stress power under superimposed rigid body motions. The third-order stress tensor $\mathbf{T}_{3}$ enjoys the same symmetry in the last two entries as the second velocity gradient, since an antimetric part would give no contribution to the virtual power. The virtual stress power of a whole body may be reformulated by twice applying the divergence theorem:

$$
\begin{aligned}
\delta \Pi_{i} & =\int\left(\mathbf{T}_{2}: \delta \mathbf{v} \otimes \nabla+\mathbf{T}_{3}: \cdot \delta \mathbf{v} \otimes \nabla \otimes \nabla\right) \mathrm{d} V \\
& =-\int \delta \mathbf{v} \cdot \mathbf{X} \cdot \nabla \mathrm{d} V+\int\left(\delta \mathbf{v} \cdot \mathbf{X} \cdot \mathbf{n}+\left(\mathbf{T}_{3} \cdot \mathbf{n}\right): \delta \mathbf{v} \otimes \nabla\right) \mathrm{d} A
\end{aligned}
$$

We used the abbreviation

$$
\mathbf{X} \equiv \mathbf{T}_{2}-\mathbf{T}_{3} \cdot \nabla
$$

Imagining any cut through a body, we infer from the surface integral that the interaction across such a cut is described by the vector $\mathbf{X} \cdot \mathbf{n}$ and the non-symmetric second-order tensor $\mathbf{T}_{3} \cdot \mathbf{n}$. Both values depend on the special cut only through the local orientation $\mathbf{n}$ as is the case with Cauchy's stress vector in the classical theory. This interpretation differs from that usually found in the literature (cf. $[3,7-11]$ ) and has serious consequences in the sequel.

If we want to treat boundary conditions it is useful to split the gradient on the surface into a tangential and a normal part.

$$
\nabla=\nabla_{t}+\mathbf{n} \frac{\partial}{\partial n}
$$

and to introduce the non-symmetric second-order tensor

$$
\mathbf{T} \equiv\left(\mathbf{T}_{3} \cdot \mathbf{n}\right) \cdot(\mathbf{1}-\mathbf{n} \otimes \mathbf{n})
$$

This allows the following representation of the surface integral

$$
\int \delta \mathbf{v} \cdot \mathbf{X} \cdot \mathbf{n} \mathrm{d} A+\int \mathbf{T}: \delta \mathbf{v} \otimes \nabla_{t} \mathrm{~d} A+\int \frac{\partial \delta \mathbf{v}}{\partial n} \cdot \mathbf{T}_{3}: \mathbf{n} \otimes \mathbf{n} \mathrm{d} A
$$

The virtual power of the external forces is given by

$$
\delta \Pi_{e}=\int \delta \mathbf{v} \cdot \rho(\mathbf{b}-\mathbf{a}) \mathrm{d} V+\int \delta \mathbf{v} \cdot \mathbf{t}_{e} \mathrm{~d} A
$$




$$
+\int \frac{\partial \delta \mathbf{v}}{\partial n} \cdot \overline{\mathbf{t}}_{e} \mathrm{~d} A+\int \mathbf{T}_{e}: \delta \mathbf{v} \otimes \nabla_{t} \mathrm{~d} A
$$

The mass density is denoted by $\rho$, the body force per unit mass by $\mathbf{b}$, the acceleration with respect to an inertial frame by $\mathbf{a}$ and the stress on the surface by $\mathbf{t}_{e}$. Additional surface stresses $\overline{\mathbf{t}}_{e}$ and $\mathbf{T}_{e}$ are possible with second gradient materials. The necessity of $\mathbf{T}_{e}$ was overlooked so far.

The equality of the internal and external virtual power requires

$$
\begin{aligned}
0= & -\int \delta \mathbf{v} \cdot(\mathbf{X} \cdot \nabla+\rho(\mathbf{b}-\mathbf{a})) \mathrm{d} V+\int \frac{\partial \delta \mathbf{v}}{\partial n} \cdot\left(\mathbf{T}_{3}: \mathbf{n} \otimes \mathbf{n}-\overline{\mathbf{t}}_{e}\right) \mathrm{d} A \\
& +\int \delta \mathbf{v} \cdot\left(\mathbf{X} \cdot \mathbf{n}-\mathbf{t}_{e}\right) \mathrm{d} A+\int\left(\mathbf{T}-\mathbf{T}_{e}\right): \delta \mathbf{v} \otimes \nabla_{t} \mathrm{~d} A
\end{aligned}
$$

This implies the balance of momentum in the form

$$
\mathbf{X} \cdot \nabla+\rho(\mathbf{b}-\mathbf{a})=\mathbf{0}
$$

Now let us consider a boundary where the velocity $\mathbf{v}$ is given, but the normal derivative $\partial \mathbf{v} / \partial n$ of the velocity is free. So $\delta \mathbf{v}=\mathbf{0}$ and $\delta \mathbf{v} \otimes \nabla_{t}=\mathbf{0}$ and the following boundary condition remains:

$$
\mathbf{T}_{3}: \mathbf{n} \otimes \mathbf{n}=\overline{\mathbf{t}}_{e}
$$

Next we consider a free surface. It is impossible to postulate that the three independent integrals vanish since only two boundary conditions are admissible. Such a difficulty is well known from Kirchhoff's theory of plates. Thomson and Tait solved the problem by applying integration by parts along the free edge. We use Stokes' theorem

$$
\oint d \mathbf{r}=\int \mathrm{d} A \mathbf{n} \times \nabla=\int \mathrm{d} A \mathbf{n} \times \nabla_{t}
$$

in an analogous manner. We introduce the line element $d \mathbf{r}=\operatorname{td} L$ on the boundary of a smooth part of that surface and the outward unit normal there in the tangential plane $\mathbf{m}=\mathbf{t} \times \mathbf{n}$. We denote the difference of the tensors $\mathbf{T}$ and $\mathbf{T}_{e}$ on the inside and the outside of the free surface by

$$
\mathbf{S} \equiv \mathbf{T}-\mathbf{T}_{e} \text { with } \mathbf{S} \cdot \mathbf{n}=0
$$

We apply the product rule and find

$$
\begin{aligned}
\oint \delta \mathbf{v} \cdot \mathbf{S} \cdot \mathbf{m} \mathrm{d} L= & \oint \delta \mathbf{v} \cdot \mathbf{S} \cdot(d \mathbf{r} \times \mathbf{n})=\int \mathrm{d} A \delta \mathbf{v} \cdot \mathbf{S} \cdot\left(\left(\mathbf{n} \times \nabla_{t}\right) \times \mathbf{n}\right) \\
= & \int \mathrm{d} A \delta \mathbf{v} \cdot \mathbf{S} \cdot\left(\left(\mathbf{n} \times \nabla_{t}\right) \times \dot{\mathbf{n}}\right)+\int \mathrm{d} A \delta \dot{\mathbf{v}} \cdot \mathbf{S} \cdot\left(\left(\mathbf{n} \times \nabla_{t}\right) \times \mathbf{n}\right) \\
& +\int \mathrm{d} A \delta \mathbf{v} \cdot \dot{\mathbf{S}} \cdot\left(\left(\mathbf{n} \times \nabla_{t}\right) \times \mathbf{n}\right) \\
= & -\int \mathrm{d} A \delta \mathbf{v} \cdot\left(\dot{\mathbf{n}} \cdot \nabla_{t}\right) \underline{\mathbf{S} \cdot \mathbf{n}}+\int \mathrm{d} A \mathbf{S}: \delta \dot{\mathbf{v}} \otimes \nabla_{t}+\int \mathrm{d} A \delta \mathbf{v} \cdot \dot{\mathbf{S}} \cdot \nabla_{t}
\end{aligned}
$$

The accent indicates on which field $\nabla_{t}$ is to be applied. We made use of the identities

$$
\left(\mathbf{n} \times \nabla_{t}\right) \times \mathbf{n}=(\mathbf{n} \cdot \mathbf{n}) \nabla_{t}-\left(\underline{\mathbf{n} \cdot \nabla_{t}}\right) \mathbf{n}=\nabla_{t}
$$

and

$$
\left(\mathbf{n} \times \nabla_{t}\right) \times \underline{\mathbf{n}}=\underline{(\mathbf{n} \cdot \mathbf{n}) \nabla_{t}}-\left(\dot{\mathbf{n}} \cdot \nabla_{t}\right) \mathbf{n}
$$

The underlined terms vanish. So the local mean curvature $H=-\mathbf{n} \cdot \nabla_{t} / 2$ does not enter the expression.

The last two integrals of Eq. (8) can now be replaced by

$$
\int \delta \mathbf{v} \cdot\left(\mathbf{X} \cdot \mathbf{n}-\mathbf{S} \cdot \nabla_{t}-\mathbf{t}_{e}\right) \mathrm{d} A+\oint \delta \mathbf{v} \cdot \mathbf{S} \cdot \mathbf{m} \mathrm{d} L
$$

and the following two boundary conditions remain:

$$
\mathbf{T}_{3}: \mathbf{n} \otimes \mathbf{n}=\overline{\mathbf{t}}_{e}, \quad \mathbf{S} \cdot \nabla_{t}-\mathbf{X} \cdot \mathbf{n}+\mathbf{t}_{e}=\mathbf{0}
$$


The last equation is well known. It is the equilibrium condition of forces of a thin shell with stress tensor $\mathbf{S}$. We infer that a crust shell is present at the free surface of any second gradient body. This shell is loaded from the inside by the stress $-\mathbf{X} \cdot \mathbf{n}$ and from the outside by the given stress $\mathbf{t}_{e}$. The tensor $\mathbf{S}$ may be called the surface tension of the body. Imagining any cut through the crust shell normal to the vector $\mathbf{m}$ we find a vector $\mathbf{S} \cdot \mathbf{m}$ of dimension force per length. It possesses components in the normal, shear and transverse direction. The crust shell is however free of bending and torsional moments and no surface energy is attributed to it.

The situation at a fixed boundary is ambiguous. If the counterpart of the body under consideration also consists of a second gradient material, then we learn from (8) that it can provide a reaction vector $\mathbf{t}_{e}=\mathbf{X} \cdot \mathbf{n}$ and a reaction tensor $\mathbf{T}_{e}=\mathbf{T}$ so that $\mathbf{S}=\mathbf{0}$ according to (12) and no crust shell is needed. If, on the other hand, the counterpart consists of a simple material, then it cannot provide a reaction tensor $\mathbf{T}_{e}$ and a crust shell with $\mathbf{S}=\mathbf{T}$ must exist. The reaction vector is then $\mathbf{t}_{e}=\mathbf{X} \cdot \mathbf{n}-\mathbf{S} \cdot \nabla_{t}$. This seems not to have been discussed yet.

\section{The compressible fluid}

\subsection{Kinematics}

The first velocity gradient can be split into a deviatoric and a spherical part according to

$$
\mathbf{v} \otimes \nabla=(\mathbf{v} \otimes \nabla)^{*}+\frac{1}{3}(\nabla \cdot \mathbf{v}) \mathbf{1} \text { with }(\mathbf{v} \otimes \nabla)^{*}: \mathbf{1}=0
$$

This implies the following decomposition of the second gradient:

$$
\mathbf{v} \otimes \nabla \otimes \nabla=(\mathbf{v} \otimes \nabla)^{*} \otimes \nabla+\frac{1}{3} \mathbf{1} \otimes \nabla(\nabla \cdot \mathbf{v})
$$

and hence

$$
\nabla \otimes \mathbf{v} \otimes \nabla=(\nabla \otimes \mathbf{v})^{*} \otimes \nabla+\frac{1}{3} \mathbf{1} \otimes \nabla(\nabla \cdot \mathbf{v})
$$

and

$$
\nabla \otimes \nabla \otimes \mathbf{v}=\nabla \otimes(\nabla \otimes \mathbf{v})^{*}+\frac{1}{3} \nabla(\nabla \cdot \mathbf{v}) \otimes \mathbf{1}
$$

Note that $\mathbf{v} \otimes \nabla \otimes \nabla$ enjoys a symmetry in the second and last entry, while this is not the case with $(\mathbf{v} \otimes \nabla)^{*} \otimes \nabla$. The equation of continuity reads

$$
\rho^{\bullet} \equiv \frac{\partial \rho}{\partial t}+\mathbf{v} \cdot(\nabla \rho)=-\rho(\nabla \cdot \mathbf{v})
$$

where the dot denotes the material time derivative. Therefore, we have

$$
\begin{aligned}
\left(|\nabla \rho|^{2}\right)^{\bullet} & =2|\nabla \rho||\nabla \rho|^{\bullet}=(\nabla \rho \cdot \nabla \rho)^{\bullet} \\
& =2 \nabla \rho \cdot(\nabla \rho)^{\bullet} \Longrightarrow|\nabla \rho|^{\bullet}=\mathbf{e} \cdot(\nabla \rho)^{\bullet} \quad \text { with } \mathbf{e} \equiv \frac{\nabla \rho}{|\nabla \rho|} \\
(\nabla \rho)^{\bullet} & =\frac{\partial \nabla \rho}{\partial t}+\mathbf{v} \cdot \nabla \otimes \nabla \rho=\nabla\left(\rho^{\bullet}-\mathbf{v} \cdot \nabla \rho\right)+\mathbf{v} \cdot \nabla \otimes \nabla \rho=\nabla \rho \bullet \nabla \otimes \mathbf{v} \cdot \nabla \rho \\
& =-\nabla \rho \nabla \cdot \mathbf{v}-\rho \nabla(\nabla \cdot \mathbf{v})-\nabla \otimes \mathbf{v} \cdot \nabla \rho \\
& =-\frac{4}{3} \nabla \rho \nabla \cdot \mathbf{v}-\rho \nabla(\nabla \cdot \mathbf{v})-(\nabla \otimes \mathbf{v})^{*} \cdot \nabla \rho
\end{aligned}
$$

and

$$
\begin{aligned}
& |\nabla \rho|^{\bullet}=-|\nabla \rho|(\nabla \cdot \mathbf{v}+\mathbf{e} \cdot \nabla \otimes \mathbf{v} \cdot \mathbf{e})-\rho \mathbf{e} \cdot \nabla(\nabla \cdot \mathbf{v}) \\
& \nabla \cdot \mathbf{v}=-\frac{\rho}{\rho}, \quad \nabla(\nabla \cdot \mathbf{v})=-\frac{1}{\rho}(\nabla \rho)^{\bullet}-\frac{1}{\rho}(\nabla \otimes \mathbf{v})^{*} \cdot \nabla \rho+\frac{4 \rho^{\bullet}}{3 \rho^{2}} \nabla \rho
\end{aligned}
$$




\subsection{Viscous stresses}

First, we deal with a linear compressible material. Its viscous stresses of second and third order are given by the full set of hemitropic functions of the first and second velocity gradient ( cf. [10], Eqs. (96), (97), where $\alpha_{8}=\alpha_{9}$ in our notation because the stresses are derived from a dissipation potential):

$$
\begin{aligned}
\mathbf{T}_{2 v}= & \operatorname{sym}\left[\alpha_{1}(\nabla \cdot \mathbf{v}) \mathbf{1}+\alpha_{2} \mathbf{v} \otimes \nabla+\alpha_{8} \boldsymbol{\epsilon}: \mathbf{v} \otimes \nabla \otimes \nabla\right] \\
\mathbf{T}_{3 v}= & \operatorname{sym}^{[23]}\left[\alpha_{3} \mathbf{1} \otimes \nabla(\nabla \cdot \mathbf{v})+\frac{\alpha_{4}}{2}(\nabla(\nabla \cdot \mathbf{v}) \otimes \mathbf{1}+\mathbf{1} \otimes \Delta \mathbf{v})\right. \\
& \left.+\alpha_{5} \Delta \mathbf{v} \otimes \mathbf{1}+\alpha_{6} \mathbf{v} \otimes \nabla \otimes \nabla+\alpha_{7} \nabla \otimes \mathbf{v} \otimes \nabla+\alpha_{9} \boldsymbol{\epsilon} \cdot \operatorname{sym}[\mathbf{v} \otimes \nabla]\right]
\end{aligned}
$$

Here, $\boldsymbol{\epsilon}$ denotes the third-order permutation tensor. The notation [23] indicates that the symmetry is meant with respect to the second and third entry. Introducing the results of the last subsection we separate the contributions of isochoric flow and volume change:

$$
\begin{aligned}
\mathbf{T}_{2 v}= & \operatorname{sym}\left[-\left(\alpha_{1}+\frac{\alpha_{2}}{3}\right) \frac{\rho^{\bullet}}{\rho} \mathbf{1}+\alpha_{2}(\mathbf{v} \otimes \nabla)^{*}+\alpha_{8} \boldsymbol{\epsilon}:(\mathbf{v} \otimes \nabla)^{*} \otimes \nabla\right] \\
\mathbf{T}_{3 v}= & \operatorname{sym}^{[23]}\left[\frac{\alpha_{4}}{2} \mathbf{1} \otimes(\mathbf{v} \otimes \nabla)^{*} \cdot \nabla+\alpha_{5}(\mathbf{v} \otimes \nabla)^{*} \cdot \nabla \otimes \mathbf{1}+\alpha_{6}(\mathbf{v} \otimes \nabla)^{*} \otimes \nabla\right. \\
& +\alpha_{7}(\nabla \otimes \mathbf{v})^{*} \otimes \nabla+\left(\alpha_{3}+\frac{\alpha_{4}}{6}+\frac{\alpha_{7}}{3}+\frac{\alpha_{6}}{3}\right) \mathbf{1} \otimes\left(-\frac{1}{\rho}(\nabla \rho)^{\bullet}-\frac{1}{\rho}(\nabla \otimes \mathbf{v})^{*} \cdot \nabla \rho+\frac{4 \rho^{\bullet}}{3 \rho^{2}} \nabla \rho\right) \\
& \left.+\left(\frac{\alpha_{4}}{2}+\frac{\alpha_{5}}{3}\right)\left(-\frac{1}{\rho}(\nabla \rho)^{\bullet}-\frac{1}{\rho}(\nabla \otimes \mathbf{v})^{*} \cdot \nabla \rho+\frac{4 \rho^{\bullet}}{3 \rho^{2}} \nabla \rho\right) \otimes \mathbf{1}+\alpha_{9} \boldsymbol{\epsilon} \cdot \operatorname{sym}[\mathbf{v} \otimes \nabla]^{*}\right]
\end{aligned}
$$

\subsection{Elastic stresses}

A material that is completely characterized by the constitutive laws of the last subsection would have a strange behavior: Given a constant state of stress, it could change its volume without limit. Since this is not realistic, we assume, that a deviation of the mass density $\rho$ from some value $\rho_{0}$ is accompanied by a storage of elastic energy. Since we deal with second gradient materials, the strain energy per unit mass should not only depend on the local mass density $\rho$ but also on its spatial gradient $\nabla \rho$. However, the principle of invariance under superimposed rigid body modifications requires that not the direction but only the magnitude $|\nabla \rho|$ of this gradient is an admissible variable:

$$
w=w(\rho,|\nabla \rho|), \quad w^{\bullet}=\frac{\partial w}{\partial \rho} \rho^{\bullet}+\frac{\partial w}{\partial|\nabla \rho|}|\nabla \rho|^{\bullet}
$$

The power of the elastic stresses per unit volume is then

$$
\begin{aligned}
\pi_{e} & =\mathbf{T}_{2 e}: \mathbf{v} \otimes \nabla+\mathbf{T}_{3 e}: \cdot \mathbf{v} \otimes \nabla \otimes \nabla=\rho w^{\bullet} \\
& =-\frac{\partial w}{\partial \rho} \rho^{2} \nabla \cdot \mathbf{v}-\frac{\partial w}{\partial|\nabla \rho|} \rho(|\nabla \rho|(\nabla \cdot \mathbf{v}+\mathbf{e} \cdot \nabla \otimes \mathbf{v} \cdot \mathbf{e})+\rho \mathbf{e} \cdot \nabla(\nabla \cdot \mathbf{v}))
\end{aligned}
$$

We infer

$$
\begin{aligned}
& \mathbf{T}_{2 e}=-\left(\frac{\partial w}{\partial \rho} \rho^{2}+\frac{\partial w}{\partial|\nabla \rho|} \rho|\nabla \rho|\right) \mathbf{1}-\frac{\partial w}{\partial|\nabla \rho|} \rho|\nabla \rho| \mathbf{e} \otimes \mathbf{e} \\
& \mathbf{T}_{3 e}=-\frac{\partial w}{\partial|\nabla \rho|} \rho^{2} \operatorname{sym}^{[23]}[\mathbf{1} \otimes \mathbf{e}]
\end{aligned}
$$

The fluid is assumed to be materially uniform and free of stress at the mass density $\rho_{0}$. We restrict our attention to the case where $\left|\rho-\rho_{0}\right|$ and $|\nabla \rho|$ are small, so that the following quadratic form is appropriate:

$$
w=\frac{K}{2 \rho_{0}^{3}}\left(\left(\rho-\rho_{0}\right)^{2}+L^{2}|\nabla \rho|^{2}+2 \beta L\left(\rho-\rho_{0}\right)|\nabla \rho|\right)
$$


Here, $K>0$ denotes the modulus of compressibility, $L$ a characteristic elastic length of the gradient material and $\beta$ a dimensionless parameter, which must satisfy $|\beta|<1$ in order to make the strain energy positive definite. We obtain the following elastic stresses:

$$
\begin{aligned}
& \mathbf{T}_{2 e}=-\underbrace{\left(\frac{\rho}{\rho_{0}}\right)^{2}} \frac{K}{\rho_{0}}\left(\rho-\rho_{0}+\beta L|\nabla \rho|\right) \mathbf{1}-\underline{\left(\frac{\rho}{\rho_{0}}\right) \frac{K}{\rho_{0}}\left(\beta\left(\rho-\rho_{0}\right)+L|\nabla \rho|\right) \frac{L|\nabla \rho|}{\rho_{0}}(\mathbf{1}+\mathbf{e} \otimes \mathbf{e})} \\
& \mathbf{T}_{3 e}=-\underbrace{\left(\frac{\rho}{\rho_{0}}\right)^{2} \frac{K}{\rho_{0}}}\left(\beta\left(\rho-\rho_{0}\right)+L|\nabla \rho|\right) L \operatorname{sym}^{[23]}[\mathbf{1} \otimes \mathbf{e}]
\end{aligned}
$$

The underlined expression is of a smaller than the first order and shall be neglected. Moreover, we can replace the underbraced expressions by 1 . So we arrive at

$$
\begin{aligned}
& \mathbf{T}_{2 e}=-\frac{K}{\rho_{0}}\left(\rho-\rho_{0}+\beta L|\nabla \rho|\right) \mathbf{1} \\
& \mathbf{T}_{3 e}=-\frac{K L^{2}}{\rho_{0}}\left(\beta \frac{\rho-\rho_{0}}{L|\nabla \rho|}+1\right) \operatorname{sym}^{[23]}[\mathbf{1} \otimes \nabla \rho]
\end{aligned}
$$

\subsection{Total stresses}

We choose a parallel connection of the elastic and viscous stresses in the sense of Kelvin-Voigt:

$$
\mathbf{T}_{2}=\mathbf{T}_{2 v}+\mathbf{T}_{2 e}, \quad \mathbf{T}_{3}=\mathbf{T}_{3 v}+\mathbf{T}_{3 e}
$$

Moreover, we define the scalar field

$$
u \equiv \frac{K}{\rho_{0}}\left(\rho-\rho_{0}\right)
$$

and the two retardation times corresponding to the second- and third-order constitutive equation:

$$
t_{2} \equiv \frac{\alpha_{1}}{K}, \quad t_{3} \equiv \frac{\alpha_{3}}{K L^{2}}
$$

The denominators $\rho$ in the viscous stresses are replaced by $\rho_{0}$. So we arrive at

$$
\begin{aligned}
\mathbf{T}_{2}= & \operatorname{sym}\left[\alpha_{2}(\mathbf{v} \otimes \nabla)^{*}+\alpha_{8} \boldsymbol{\epsilon}:(\mathbf{v} \otimes \nabla)^{*} \otimes \nabla\right]-\left(\left(\frac{\alpha_{2}}{3 K}+t_{2}\right) u^{\bullet}+u+\beta L|\nabla u|\right) \mathbf{1} \\
\mathbf{T}_{3}= & \operatorname{sym}^{[23]}\left[\frac{\alpha_{4}}{2} \mathbf{1} \otimes(\mathbf{v} \otimes \nabla)^{*} \cdot \nabla+\alpha_{5}(\mathbf{v} \otimes \nabla)^{*} \cdot \nabla \otimes \mathbf{1}\right. \\
& +\alpha_{6}(\mathbf{v} \otimes \nabla)^{*} \otimes \nabla+\alpha_{7}(\nabla \otimes \mathbf{v})^{*} \otimes \nabla \\
& +\mathbf{1} \otimes L^{2}\left(( t _ { 3 } + \frac { 1 } { K L ^ { 2 } } ( \frac { \alpha _ { 4 } } { 6 } + \frac { \alpha _ { 7 } } { 3 } + \frac { \alpha _ { 6 } } { 3 } ) ) \left(-(\nabla u)^{\bullet}-(\nabla \otimes \mathbf{v})^{*} \cdot \nabla u\right.\right. \\
& \left.\left.+\frac{4}{3 K} u^{\bullet} \nabla u\right)-\beta u \frac{\nabla u}{L|\nabla u|}-\nabla u\right) \\
& \left.+\frac{1}{K}\left(\frac{\alpha_{4}}{2}+\frac{\alpha_{5}}{3}\right)\left(-(\nabla u)^{\bullet}-(\nabla \otimes \mathbf{v})^{*} \cdot \nabla u+\frac{4}{3 K} u^{\bullet} \nabla u\right) \otimes \mathbf{1}+\alpha_{9} \boldsymbol{\epsilon} \cdot \operatorname{sym}[\mathbf{v} \otimes \nabla]^{*}\right]
\end{aligned}
$$




\section{The incompressible fluid}

Classical approach We apply the constraint

$$
\rho \equiv \rho_{0} \quad \Longrightarrow \quad \nabla \cdot \mathbf{v} \equiv 0, \quad \nabla(\nabla \cdot \mathbf{v}) \equiv \mathbf{0}, \quad(\mathbf{v} \otimes \nabla)^{*}=\mathbf{v} \otimes \nabla, \quad(\mathbf{v} \otimes \nabla)^{*} \cdot \nabla=\Delta \mathbf{v}
$$

So no elastic energy $w$ and no elastic stresses are needed. The viscous stresses reduce to

$$
\begin{aligned}
\mathbf{T}_{2 D}= & \operatorname{sym}\left[\alpha_{2} \mathbf{v} \otimes \nabla+\alpha_{8} \boldsymbol{\epsilon}: \mathbf{v} \otimes \nabla \otimes \nabla\right] \\
\mathbf{T}_{3 D}= & \operatorname{sym}^{[23]}\left[\frac{\alpha_{4}}{2} \mathbf{1} \otimes \Delta \mathbf{v}+\alpha_{5} \Delta \mathbf{v} \otimes \mathbf{1}+\alpha_{6} \mathbf{v} \otimes \nabla \otimes \nabla\right. \\
& \left.+\alpha_{7} \nabla \otimes \mathbf{v} \otimes \nabla+\alpha_{9} \boldsymbol{\epsilon} \cdot \operatorname{sym}[\mathbf{v} \otimes \nabla]\right]
\end{aligned}
$$

These stresses are endowed with the suffix $D$, because they are determined by the isochoric flow field. But reaction stresses will also be present that do no work during any isochoric motion, namely

$$
\mathbf{T}_{2 R}=-p \mathbf{1}, \quad \mathbf{T}_{3 R}=\operatorname{sym}^{[23]}[\mathbf{1} \otimes 2 \mathbf{q}]
$$

Indeed

$$
\begin{aligned}
\mathbf{T}_{2 R}: \mathbf{v} \otimes \nabla+\mathbf{T}_{3 R}: \cdot \mathbf{v} \otimes \nabla \otimes \nabla & =-p \mathbf{1}: \mathbf{v} \otimes \nabla+\mathbf{1} \otimes 2 \mathbf{q}: \cdot \mathbf{v} \otimes \nabla \otimes \nabla \\
& =-p \nabla \cdot \mathbf{v}+2 \mathbf{q} \cdot \nabla(\nabla \cdot \mathbf{v}) \equiv 0
\end{aligned}
$$

We take note of the fact that the determined stress contribution with the parameter $\alpha_{4}$ does no work during any motion. So we may choose $\alpha_{4}=0$ and adapt the vector $\mathbf{q}$. If we want to treat some boundary value problem, we are confronted by the unknown scalar field $p$ and the vector fields $\mathbf{v}$ and $\mathbf{q}$. In general there is no definite solution to such a problem. Therefore, we need a better description which will be presented in what follows.

Advanced approach There will hardly be any real material that is totally incompressible. However, the modulus of compressibility $K$ may be very high so that only small changes of mass density will occur. Such a behavior has been described in the foregoing subsection. We may go now one step further and let $K$ tend toward infinity. The stresses and hence the values of $u$ can only remain finite if $K \rightarrow \infty$ implies $\rho \rightarrow \rho_{0}$. We obtain

$$
\begin{aligned}
\mathbf{T}_{2}= & \operatorname{sym}\left[\alpha_{2}(\mathbf{v} \otimes \nabla)+\underline{\alpha_{8} \boldsymbol{\epsilon}:(\mathbf{v} \otimes \nabla) \otimes \nabla}\right]-p \mathbf{1} \\
\mathbf{T}_{3}= & \operatorname{sym}^{[23]}\left[\frac{\alpha_{4}}{2} \mathbf{1} \otimes \Delta \mathbf{v}+\alpha_{5} \Delta \mathbf{v} \otimes \mathbf{1}+\alpha_{6} \mathbf{v} \otimes \nabla \otimes \nabla\right. \\
& \left.+\alpha_{7} \nabla \otimes \mathbf{v} \otimes \nabla+\underline{\alpha_{9} \boldsymbol{\epsilon} \cdot \operatorname{sym}[\mathbf{v} \otimes \nabla]}+\mathbf{1} \otimes 2 \mathbf{q}\right]
\end{aligned}
$$

with

$$
\begin{aligned}
p & =t_{2} u^{\bullet}+u+\beta L|\nabla u| \\
2 \mathbf{q} & =-L^{2}\left(t_{3}\left((\nabla u)^{\bullet}+(\nabla \otimes \mathbf{v}) \cdot \nabla u\right)+\beta u \frac{\nabla u}{L|\nabla u|}+\nabla u\right)
\end{aligned}
$$

If we compare this with the total stresses $\mathbf{T}_{2 D}+\mathbf{T}_{2 R}$ and $\mathbf{T}_{3 D}+\mathbf{T}_{3 R}$ of the classical approach, we find a perfect formal agreement. Such an agreement was already discovered in 1980 by Bertram [12] in the context of simple materials. However, our result for second gradient fluids goes far beyond this finding. Here, the fields $p$ and $\mathbf{q}$ do not represent four unknown scalar functions but are derived from only one scalar function $u$. This makes it possible to obtain information on the internal stresses, the surface tension and the forces acting on fixed boundaries that is not available with the classical approach. The value of $\alpha_{4}$ is not arbitrary in this context but gives rise to an independent stress contribution.

Note We study strictly isochoric motions. No viscoelastic volume change is allowed. Nevertheless, the limit from the weakly compressible case allows the reduction to one function $u$. Its contribution to the stresses makes use of four viscoelastic constants $L, \beta, t_{2}, t_{3}$ which describe the behavior under volumetric changes, although these changes were eliminated by our limiting process.

If $L=0$ is assumed, then the volumetric behavior is that of a simple material and $\mathbf{q} \equiv \mathbf{0}$ is valid. 


\section{Admissible ranges of the material constants}

The remainder of this paper is restricted to materials with $\alpha_{8}=\alpha_{9}=0$. So the underlined expressions in the constitutive law (50), (51) are canceled and there is no coupling between the first and second gradient. Only hemitropic material functions that are also isotropic remain. The stress power per unit volume of our incompressible fluid then can be represented as follows:

$$
\begin{aligned}
\pi_{i}= & \mathbf{T}_{2}: \mathbf{v} \otimes \nabla+\mathbf{T}_{3}: \cdot \mathbf{v} \otimes \nabla \otimes \nabla \\
= & \operatorname{sym}\left[\alpha_{2} \mathbf{v} \otimes \nabla\right]: \mathbf{v} \otimes \nabla+\operatorname{sym}^{[23]}\left[\alpha_{5} \Delta \mathbf{v} \otimes \mathbf{1}+\alpha_{6} \mathbf{v} \otimes \nabla \otimes \nabla+\alpha_{7} \nabla \otimes \mathbf{v} \otimes \nabla\right]: \cdot \mathbf{v} \otimes \nabla \otimes \nabla \\
= & \alpha_{2} \operatorname{sym}[\mathbf{v} \otimes \nabla]: \operatorname{sym}[\mathbf{v} \otimes \nabla]+\left(\alpha_{5}+\frac{1}{10}\left(4 \alpha_{6}-\alpha_{7}\right)\right) \Delta \mathbf{v} \cdot \Delta \mathbf{v} \\
& +\frac{2}{9}\left(2 \alpha_{6}-\alpha_{7}\right)\left(\mathbf{v} \otimes \nabla \otimes \nabla-\frac{1}{2} \Delta \mathbf{v} \otimes \mathbf{1}-\operatorname{sym}^{[23]}\left[\nabla \otimes \mathbf{v} \otimes \nabla-\frac{1}{2} \mathbf{1} \otimes \Delta \mathbf{v}\right]\right) \\
& \quad:\left(\mathbf{v} \otimes \nabla \otimes \nabla-\frac{1}{2} \Delta \mathbf{v} \otimes \mathbf{1}-\operatorname{sym}^{[23]}\left[\nabla \otimes \mathbf{v} \otimes \nabla-\frac{1}{2} \mathbf{1} \otimes \Delta \mathbf{v}\right]\right) \\
+ & \frac{1}{9}\left(\alpha_{6}+\alpha_{7}\right)\left(\mathbf{v} \otimes \nabla \otimes \nabla-\frac{1}{5} \Delta \mathbf{v} \otimes \mathbf{1}+2 \operatorname{sym}^{[23]}\left[\nabla \otimes \mathbf{v} \otimes \nabla-\frac{1}{5} \mathbf{1} \otimes \Delta \mathbf{v}\right]\right) \\
& : \cdot\left(\mathbf{v} \otimes \nabla \otimes \nabla-\frac{1}{5} \Delta \mathbf{v} \otimes \mathbf{1}+2 \operatorname{sym}^{[23]}\left[\nabla \otimes \mathbf{v} \otimes \nabla-\frac{1}{5} \mathbf{1} \otimes \Delta \mathbf{v}\right]\right)
\end{aligned}
$$

These reformulations are based on the identities

$$
\mathbf{v} \otimes \nabla \otimes \nabla: \cdot \mathbf{v} \otimes \nabla \otimes \nabla=\nabla \otimes \mathbf{v} \otimes \nabla: \cdot \nabla \otimes \mathbf{v} \otimes \nabla=\nabla \otimes \nabla \otimes \mathbf{v}: \cdot \nabla \otimes \nabla \otimes \mathbf{v}
$$

and

$$
\mathbf{v} \otimes \nabla \otimes \nabla: \cdot \nabla \otimes \mathbf{v} \otimes \nabla=\mathbf{v} \otimes \nabla \otimes \nabla: \cdot \nabla \otimes \nabla \otimes \mathbf{v}=\nabla \otimes \mathbf{v} \otimes \nabla: \cdot \nabla \otimes \nabla \otimes \mathbf{v}
$$

The stress power is fully dissipated. This implies that the coefficients of the four quadratic expressions must not be negative. So we obtain the following restrictions on the material constants:

$$
\alpha_{2} \geq 0, \quad \alpha_{5}+\frac{1}{10}\left(4 \alpha_{6}-\alpha_{7}\right) \geq 0, \quad-\alpha_{6} \leq \alpha_{7} \leq 2 \alpha_{6} \quad \Longrightarrow \quad \alpha_{6} \geq 0
$$

However, it is necessary to prove that the three expressions that contain second derivatives of $\mathbf{v}$ are independent of each other. The choice

$$
\mathbf{v} \otimes \nabla \otimes \nabla=\operatorname{sym}^{[23]}[\nabla \otimes \mathbf{v} \otimes \nabla]=\mathbf{e}_{1} \otimes\left(\mathbf{e}_{1} \otimes \mathbf{e}_{1}-\mathbf{e}_{2} \otimes \mathbf{e}_{2}\right)-\mathbf{e}_{2} \otimes\left(\mathbf{e}_{1} \otimes \mathbf{e}_{2}+\mathbf{e}_{2} \otimes \mathbf{e}_{1}\right)
$$

yields $\Delta \mathbf{v}=\mathbf{0}$, and only the last one of the expressions differs from zero. The choice

$$
\mathbf{v} \otimes \nabla \otimes \nabla=-2 \operatorname{sym}^{[23]}[\nabla \otimes \mathbf{v} \otimes \nabla]=\mathbf{e}_{1} \otimes\left(\mathbf{e}_{2} \otimes \mathbf{e}_{3}+\mathbf{e}_{3} \otimes \mathbf{e}_{2}\right)-\mathbf{e}_{3} \otimes\left(\mathbf{e}_{1} \otimes \mathbf{e}_{2}+\mathbf{e}_{2} \otimes \mathbf{e}_{1}\right)
$$

yields $\Delta \mathbf{v}=\mathbf{0}$, and only the second one of the expressions differs from zero. If we choose

$$
\mathbf{v} \otimes \nabla \otimes \nabla=2 \mathbf{e}_{1} \otimes \mathbf{1}-\operatorname{sym}^{[23]}\left[\mathbf{1} \otimes \mathbf{e}_{1}\right] \quad \Longrightarrow \quad \Delta \mathbf{v}=5 \mathbf{e}_{1},
$$

then only the first expression differs from zero.

No restrictions can be imposed on the material parameter $\alpha_{4}$ since it does not enter the stress power. 


\section{An example: flow between cylinders with free surface}

The fluid is contained between two concentric cylinders the axis of which is the vertical $z$-axis of a cylindrical coordinate system $\{r, \phi, z\}$. The inside and outside cylinders undergo steady state rotations with angular velocities

$$
r=r_{i}: \quad \omega_{i}=\omega \sin \epsilon, \quad r=r_{o}: \quad \omega_{o}=\omega \cos \epsilon \quad \Longrightarrow \quad \omega^{2}=\omega_{i}^{2}+\omega_{o}^{2}
$$

The case $\epsilon=\pi / 4$ induces a rigid body rotation of the fluid, while $\epsilon=\pi / 2$ describes a stirring process. The components of all fields shall be independent of $\phi$. We assume adhesion of the fluid at the walls and a free surface near the plane $z=0$. It will turn out that we must take into account a primary and a secondary flow pattern:

$$
\mathbf{v}=\mathbf{v}_{\mathrm{p}}+\mathbf{v}_{\mathrm{s}}, \quad \mathbf{v}_{\mathrm{p}}=v(r, z) \mathbf{e}_{\phi}, \quad \mathbf{v}_{\mathrm{s}}=v_{r}(r, z) \mathbf{e}_{r}+v_{z}(r, z) \mathbf{e}_{z}
$$

The first velocity gradients are

$$
\begin{aligned}
& \mathbf{v}_{\mathrm{p}} \otimes \nabla=v,{ }_{r} \mathbf{e}_{\phi} \otimes \mathbf{e}_{r}-\frac{v}{r} \mathbf{e}_{r} \otimes \mathbf{e}_{\phi}+v,_{z} \mathbf{e}_{\phi} \otimes \mathbf{e}_{z} \\
& \mathbf{v}_{\mathrm{S}} \otimes \nabla=v_{r}, \mathbf{e}_{r} \otimes \mathbf{e}_{r}+\frac{v_{r}}{r} \mathbf{e}_{\phi} \otimes \mathbf{e}_{\phi}+v_{z},{ }_{z} \mathbf{e}_{z} \otimes \mathbf{e}_{z}+v_{r},{ }_{z} \mathbf{e}_{r} \otimes \mathbf{e}_{z}+v_{z}, r \\
& \mathbf{e}_{z} \otimes \mathbf{e}_{r}
\end{aligned}
$$

The primary flow is isochoric since $\mathbf{v}_{\mathrm{p}} \cdot \nabla=\mathbf{v}_{\mathrm{p}} \otimes \nabla: \mathbf{1}=0$. The secondary flow is only isochoric if

$$
0=\mathbf{v}_{\mathrm{s}} \cdot \nabla=v_{r}, r+\frac{v_{r}}{r}+v_{z}, z
$$

is valid. This is guaranteed if we derive the velocity components from a function $y(r, z)$ :

$$
v_{r}=\frac{\partial y(r, z)}{\partial z}, \quad v_{z}=-\frac{y(r, z)}{r}-\frac{\partial y(r, z)}{\partial r} \Longleftrightarrow \mathbf{v}_{\mathrm{s}}=\mathbf{e}_{\phi} \times \frac{\nabla(r y)}{r}
$$

We introduce the abbreviation

$$
D \equiv \frac{\partial^{2}}{\partial r^{2}}+\frac{1}{r} \frac{\partial}{\partial r}-\frac{1}{r^{2}}+\frac{\partial^{2}}{\partial z^{2}}
$$

Then,

$$
\begin{aligned}
& \mathbf{v}_{\mathrm{s}} \otimes \nabla= y, r z \\
& \mathbf{e}_{r} \otimes \mathbf{e}_{r}+\frac{y, z}{r} \mathbf{e}_{\phi} \otimes \mathbf{e}_{\phi}-(y, r z \\
&+y,,_{z z} \mathbf{e}_{r} \otimes \mathbf{e}_{z}-(D y-y, z) \mathbf{e}_{z} \otimes \mathbf{e}_{z} \otimes \mathbf{e}_{r}
\end{aligned}
$$

The second velocity gradients become

$$
\begin{aligned}
\mathbf{v}_{\mathrm{p}} \otimes \nabla \otimes \nabla= & v,{ }_{r} \mathbf{e}_{\phi} \otimes \mathbf{e}_{r} \otimes \mathbf{e}_{r}-\left(\frac{v, r}{r}-\frac{v}{r^{2}}\right)\left(\mathbf{e}_{r} \otimes \mathbf{e}_{\phi} \otimes \mathbf{e}_{r}\right. \\
& \left.+\mathbf{e}_{r} \otimes \mathbf{e}_{r} \otimes \mathbf{e}_{\phi}-\mathbf{e}_{\phi} \otimes \mathbf{e}_{\phi} \otimes \mathbf{e}_{\phi}\right) \\
& +v,_{z z} \mathbf{e}_{\phi} \otimes \mathbf{e}_{z} \otimes \mathbf{e}_{z}+v,_{r z}\left(\mathbf{e}_{\phi} \otimes \mathbf{e}_{r} \otimes \mathbf{e}_{z}+\mathbf{e}_{\phi} \otimes \mathbf{e}_{z} \otimes \mathbf{e}_{r}\right) \\
& -\frac{v, z}{r}\left(\mathbf{e}_{r} \otimes \mathbf{e}_{\phi} \otimes \mathbf{e}_{z}+\mathbf{e}_{r} \otimes \mathbf{e}_{z} \otimes \mathbf{e}_{\phi}\right) \\
\mathbf{v}_{\mathrm{s}} \otimes \nabla \otimes \nabla= & y, r r z \mathbf{e}_{r} \otimes \mathbf{e}_{r} \otimes \mathbf{e}_{r}-\left(D y-y,{ }_{z z}\right){ }_{r} \mathbf{e}_{z} \otimes \mathbf{e}_{r} \otimes \mathbf{e}_{r} \\
& +\left(\frac{y, r z}{r}-\frac{y, z}{r^{2}}\right)\left(\mathbf{e}_{r} \otimes \mathbf{e}_{\phi} \otimes \mathbf{e}_{\phi}+\mathbf{e}_{\phi} \otimes \mathbf{e}_{r} \otimes \mathbf{e}_{\phi}+\mathbf{e}_{\phi} \otimes \mathbf{e}_{\phi} \otimes \mathbf{e}_{r}\right) \\
& -\frac{1}{r}\left(D y-y,,_{z z}\right) \mathbf{e}_{z} \otimes \mathbf{e}_{\phi} \otimes \mathbf{e}_{\phi} \\
& +y, z z z \mathbf{e}_{r} \otimes \mathbf{e}_{z} \otimes \mathbf{e}_{z}-(y, r z z \\
& \left.+\frac{y, z z}{r}\right) \mathbf{e}_{z} \otimes \mathbf{e}_{z} \otimes \mathbf{e}_{z}+y,{ }_{r z z}\left(\mathbf{e}_{r} \otimes \mathbf{e}_{r} \otimes \mathbf{e}_{z}+\mathbf{e}_{r} \otimes \mathbf{e}_{z} \otimes \mathbf{e}_{r}\right) \\
& -(D y-y, z z), z\left(\mathbf{e}_{z} \otimes \mathbf{e}_{r} \otimes \mathbf{e}_{z}+\mathbf{e}_{z} \otimes \mathbf{e}_{z} \otimes \mathbf{e}_{r}\right)
\end{aligned}
$$




$$
+\frac{y, z z}{r}\left(\mathbf{e}_{\phi} \otimes \mathbf{e}_{\phi} \otimes \mathbf{e}_{z}+\mathbf{e}_{\phi} \otimes \mathbf{e}_{z} \otimes \mathbf{e}_{\phi}\right)
$$

Then,

$$
\begin{aligned}
\Delta \mathbf{v}_{\mathrm{p}} & =\mathbf{v}_{\mathrm{p}} \otimes \nabla \otimes \nabla: \mathbf{1}=\Delta\left(v \mathbf{e}_{\phi}\right)=D v \mathbf{e}_{\phi} \\
\Delta \mathbf{v}_{\mathrm{s}} & =\Delta\left(\mathbf{e}_{\phi} \times \frac{\nabla(r y)}{r}\right)=D y,{ }_{z} \mathbf{e}_{r}-\left(D y, r+\frac{D y}{r}\right) \mathbf{e}_{z}=\mathbf{e}_{\phi} \times \frac{\nabla(r D y)}{r} \\
\Delta \Delta \mathbf{v}_{\mathrm{p}} & =D D v \mathbf{e}_{\phi}, \quad \Delta \Delta \mathbf{v}_{\mathrm{s}}=\mathbf{e}_{\phi} \times \frac{\nabla(r D D y)}{r}
\end{aligned}
$$

The reaction stresses are governed by

$$
p=p(r, z), \quad \mathbf{q}(r, z, \phi)=q_{r}(r, z) \mathbf{e}_{r}(\phi)+q_{\phi}(r, z) \mathbf{e}_{\phi}(\phi)+q_{z}(r, z) \mathbf{e}_{z}
$$

We apply the constitutive equations (50), (51) without the underlined terms and obtain

$$
\begin{aligned}
\mathbf{X} \equiv & \mathbf{T}_{2}-\mathbf{T}_{3} \cdot \nabla=\alpha_{2} \operatorname{sym}[\mathbf{v} \otimes \nabla]-\left(\alpha_{5}+\alpha_{6}\right) \Delta \mathbf{v} \otimes \nabla \\
& -\left(\frac{\alpha_{4}}{4}+\frac{\alpha_{7}}{2}\right) \nabla \otimes \Delta \mathbf{v}-(p+\mathbf{q} \cdot \nabla) \mathbf{1}-\nabla \otimes \mathbf{q}
\end{aligned}
$$

The shape of the free surface is characterized by

$$
z=h(r)
$$

The vectors

$$
\mathbf{g}=\mathbf{e}_{z}-\nabla h=\mathbf{e}_{z}-h^{\prime}(r) \mathbf{e}_{r}, \quad \mathbf{n}=\frac{\mathbf{g}}{|\mathbf{g}|}
$$

are normal to that surface which is free of stress.

\section{A power series solution}

Let $\epsilon$ be fixed. Then, $\mathbf{v}_{\mathrm{p}}, \mathbf{v}_{\mathrm{s}}, p, \mathbf{q}$ and $h$ depend on $\omega$ in a nonlinear manner since the acceleration is a quadratic expression of the velocities. The fields $y, p, q_{r}$ and $q_{z}$ as well as the function $h$ are even functions of $\omega$, while $v$ and $q_{\phi}$ are odd. We express all the fields as power series of $\omega$ truncated after the terms of order $\omega^{2}$ (the order symbol $o$ has the property $\left.\lim _{\omega \rightarrow 0} o\left(\omega^{2}\right) / \omega^{2}=0\right)$ :

$$
\begin{aligned}
v & =\omega v_{1}+o\left(\omega^{2}\right), \quad y=\omega^{2} y_{2}+o\left(\omega^{2}\right), \quad p=p_{0}+\omega^{2} p_{2}+o\left(\omega^{2}\right) \\
q_{r} & =q_{r 0}+\omega^{2} q_{r 2}+o\left(\omega^{2}\right), \quad q_{\phi}=\omega q_{\phi 1}+o\left(\omega^{2}\right), \quad q_{z}=q_{z 0}+\omega^{2} q_{z 2}+o\left(\omega^{2}\right) \\
h & =\omega^{2} h_{2}+o\left(\omega^{2}\right), \quad \mathbf{g}=\mathbf{e}_{z}-\omega^{2} h_{2}^{\prime} \mathbf{e}_{r}+o\left(\omega^{2}\right)
\end{aligned}
$$

and hence on the free surface

$$
|\mathbf{g}|=1+o\left(\omega^{2}\right), \quad \mathbf{n}=\frac{\mathbf{g}}{|\mathbf{g}|}=\mathbf{e}_{z}-\omega^{2} h_{2}^{\prime} \mathbf{e}_{r}+o\left(\omega^{2}\right)
$$

We will also use the notations

$$
\mathbf{X}=\mathbf{X}_{0}+\omega \mathbf{X}_{1}+\omega^{2} \mathbf{X}_{2}+o\left(\omega^{2}\right), \quad \mathbf{S}=\mathbf{S}_{0}+\omega \mathbf{S}_{1}+\omega^{2} \mathbf{S}_{2}+o\left(\omega^{2}\right)
$$

We discuss the walls $r=r_{i}$ and $r=r_{o}$ first. We need the following tensor

$$
\begin{aligned}
\mathbf{T}_{3}\left(r_{k}, \phi, z\right) \cdot \mathbf{e}_{r}= & \operatorname{sym}^{[23]}\left[\frac{\alpha_{4}}{2} \mathbf{1} \otimes \Delta \mathbf{v}+\alpha_{5} \Delta \mathbf{v} \otimes \mathbf{1}+\alpha_{6} \mathbf{v} \otimes \nabla \otimes \nabla\right. \\
& \left.+\alpha_{7} \nabla \otimes \mathbf{v} \otimes \nabla+\mathbf{1} \otimes 2 \mathbf{q}\right]\left(r_{k}, \phi, z\right) \cdot \mathbf{e}_{r}
\end{aligned}
$$




$$
\begin{aligned}
& =\left(q_{r 0} \underline{\left(2 \mathbf{e}_{r} \otimes \mathbf{e}_{r}\right.}+\mathbf{e}_{\phi} \otimes \mathbf{e}_{\phi}+\mathbf{e}_{z} \otimes \mathbf{e}_{z}\right)+q_{z 0} \mathbf{e}_{r} \otimes \mathbf{e}_{z} \\
& +\omega\left(\frac{\alpha_{4}}{4} D v_{1} \mathbf{e}_{r} \otimes \mathbf{e}_{\phi}+\underline{\alpha_{5} D v_{1} \mathbf{e}_{\phi} \otimes \mathbf{e}_{r}}\right.
\end{aligned}
$$

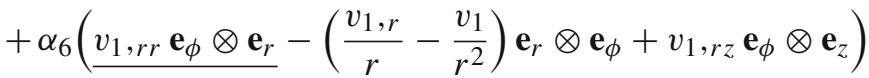

$$
\begin{aligned}
& +\frac{\alpha_{7}}{2}\left(-\left(\frac{v_{1}, r}{r}-\frac{v_{1}}{r^{2}}\right) \underline{\left(2 \mathbf{e}_{\phi} \otimes \mathbf{e}_{r}\right.}+\mathbf{e}_{r} \otimes \mathbf{e}_{\phi}\right)+v_{1, r r} \mathbf{e}_{r} \otimes \mathbf{e}_{\phi} \\
& \left.\left.+\left(v_{1}, r z-\frac{v_{1}, z}{r}\right) \mathbf{e}_{z} \otimes \mathbf{e}_{\phi}-\frac{v_{1}, z}{r} \mathbf{e}_{\phi} \otimes \mathbf{e}_{z}\right)+q_{\phi 1} \mathbf{e}_{r} \otimes \mathbf{e}_{\phi}\right) \\
& +\omega^{2}\left(\frac{\alpha_{4}}{4}\left(D y_{2}, z \underline{\left(2 \mathbf{e}_{r} \otimes \mathbf{e}_{r}\right.}+\mathbf{e}_{\phi} \otimes \mathbf{e}_{\phi}+\mathbf{e}_{z} \otimes \mathbf{e}_{z}\right)-\left(D y_{2}, r+\frac{D y_{2}}{r}\right) \mathbf{e}_{r} \otimes \mathbf{e}_{z}\right) \\
& +\alpha_{5}\left(D y_{2}, z \mathbf{e}_{r} \otimes \mathbf{e}_{r}-\left(D y_{2}, r+\frac{D y_{2}}{r}\right) \mathbf{e}_{z} \otimes \mathbf{e}_{r}\right) \\
& +\alpha_{6}\left(y_{2}, r r z \underline{\left(\mathbf{e}_{r} \otimes \mathbf{e}_{r}\right.}-\mathbf{e}_{z} \otimes \mathbf{e}_{z}\right)+\left(\frac{y_{2}, r z}{r}-\frac{y_{2}, z}{r^{2}}\right)\left(\mathbf{e}_{\phi} \otimes \mathbf{e}_{\phi}-\mathbf{e}_{z} \otimes \mathbf{e}_{z}\right) \\
& \left.\left.+y_{2}, r_{z z} \mathbf{e}_{r} \otimes \mathbf{e}_{z}-\underline{\left(D y_{2}, r\right.}-y_{2}, r z z\right) \mathbf{e}_{z} \otimes \mathbf{e}_{r}\right)
\end{aligned}
$$

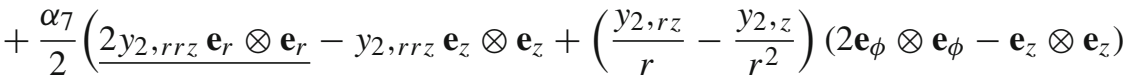

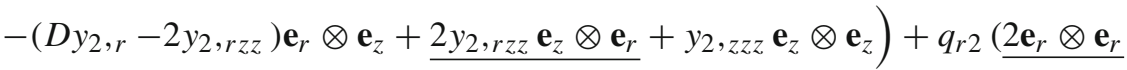

$$
\begin{aligned}
& \left.\left.\left.+\mathbf{e}_{\phi} \otimes \mathbf{e}_{\phi}+\mathbf{e}_{z} \otimes \mathbf{e}_{z}\right)+q_{z 2} \mathbf{e}_{r} \otimes \mathbf{e}_{z}\right)\right)\left(r_{k}, \phi, z\right)+o\left(\omega^{2}\right) \quad k=i, o
\end{aligned}
$$

The tensor $\mathbf{T}$ that acts on the inner wall shall be denoted by $\mathbf{T}_{i}$ and is obtained if all the underlined terms above are dropped:

$$
\mathbf{T}_{i}=\left(\mathbf{T}_{3}\left(r_{i}, \phi, z\right) \cdot \mathbf{e}_{r}\right) \cdot\left(\mathbf{e}_{\phi} \otimes \mathbf{e}_{\phi}+\mathbf{e}_{z} \otimes \mathbf{e}_{z}\right)
$$

The stress vector that acts on the inner wall is

$$
\begin{aligned}
& \mathbf{X}\left(r_{i}, \phi, z\right) \cdot \mathbf{e}_{r}=-\left(p_{0}+2 q_{r 0}, r+\frac{q_{r 0}}{r}+q_{z 0}, z\right) \mathbf{e}_{r}-q_{r 0},_{z} \mathbf{e}_{z} \\
&+\omega\left(\frac{\alpha_{2}}{2}\left(v_{1}, r-\frac{v_{1}}{r}\right)-\left(\alpha_{5}+\alpha_{6}\right) D v_{1}, r+\left(\frac{\alpha_{4}}{4}+\frac{\alpha_{7}}{2}\right) \frac{D v_{1}}{r}+\frac{q_{\phi 1}}{r}\right) \mathbf{e}_{\phi} \\
&+\omega^{2}\left(\frac{\alpha_{2}}{2}\left(2 y_{2}, r z \mathbf{e}_{r}-\left(D y_{2}-2 y_{2}, z z\right) \mathbf{e}_{z}\right)-\left(\alpha_{5}+\alpha_{6}\right)\left(D y_{2}, r z\right.\right. \\
&\left.-\left(D y_{2}, r r+\frac{D y_{2}, r}{r}-\frac{D y_{2}}{r^{2}}\right) \mathbf{e}_{z}\right) \\
&-\left(\frac{\alpha_{4}}{4}+\frac{\alpha_{7}}{2}\right)\left(D y_{2}, r z\right. \\
&\left.\mathbf{e}_{r}+D y_{2},{ }_{z z} \mathbf{e}_{z}\right) \\
&\left.-\left(p_{2}+2 q_{r 2}, r+\frac{q_{r 2}}{r}+q_{z 2}, z\right) \mathbf{e}_{r}-q_{r 2, z} \mathbf{e}_{z}\right)+o\left(\omega^{2}\right)
\end{aligned}
$$

The fluid is assumed to adhere to the walls. So the following boundary conditions at $r=r_{k}(k=i, o)$ must be valid:

$$
\begin{aligned}
& \mathbf{v}_{\mathrm{p}}\left(r_{k}, \phi, z\right)=\omega_{k} r_{k} \mathbf{e}_{\phi} \quad \Longrightarrow \quad v_{1}\left(r_{k}, \phi, z\right)=\frac{\omega_{k}}{\omega} r_{k} \\
& \mathbf{v}_{\mathrm{s}}\left(r_{k}, \phi, z\right)=\mathbf{0} \Longrightarrow y_{2}, z\left(r_{k}, z\right)=0 \Longrightarrow y_{2}\left(r_{k}, z\right)=y_{2 k}=\text { const }, \\
& y_{2}, r\left(r_{k}, z\right)=-\frac{y_{2 k}}{r_{k}}=\text { const }
\end{aligned}
$$

We will see later that we can choose $y_{2 i}=y_{2 o}=0$ so that the boundary conditions reduce to

$$
y_{2}\left(r_{k}, z\right)=0, \quad y_{2}, r\left(r_{k}, z\right)=0 \quad k=i, o
$$


Moreover, we assume that no stress $\overline{\mathbf{t}}_{e}$ is doing work on the fluid at the walls. Therefore, the following boundary condition must also hold:

$$
\begin{aligned}
\mathbf{0}=\mathbf{T}_{3}: \mathbf{e}_{r} \otimes \mathbf{e}_{r}= & 2 q_{r 0}\left(r_{k}, z\right) \mathbf{e}_{r}+\omega\left(\alpha_{5} D v_{1}+\alpha_{6} v_{1}, r_{r}-\alpha_{7}\left(\frac{v_{1}, r}{r}-\frac{v_{1}}{r^{2}}\right)\right)\left(r_{k}, z\right) \mathbf{e}_{\phi} \\
& +\omega^{2}\left(\left(\left(\frac{\alpha_{4}}{2}+\alpha_{5}\right) D y_{2}, z+\left(\alpha_{6}+\alpha_{7}\right) y_{2}, r r z+2 q_{r 2}\right) \mathbf{e}_{r}\right. \\
& \left.+\left(-\alpha_{5} \frac{D y_{2}}{r}-\left(\alpha_{5}+\alpha_{6}\right) D y_{2}, r+\underline{\left(\alpha_{6}+\alpha_{7}\right) y_{2}, r_{z z}}\right) \mathbf{e}_{z}\right)\left(r_{k}, z\right)+o\left(\omega^{2}\right)
\end{aligned}
$$

The underlined term is zero due to (88).

We are now able to evaluate the power that the inner wall applies to the fluid. We replace the virtual velocity by the actual velocity

$$
\mathbf{v}=\omega v_{1}\left(r_{i}\right) \mathbf{e}_{\phi} \Longrightarrow \mathbf{v} \otimes \nabla_{t}=-\omega \frac{v_{1}\left(r_{i}\right)}{r_{i}} \mathbf{e}_{r} \otimes \mathbf{e}_{\phi}
$$

in the surface integral of Eq. (6) and obtain

$$
\begin{aligned}
\Pi & =\int\left(\mathbf{v} \cdot \mathbf{X} \cdot \mathbf{e}_{r}+\mathbf{T}_{i}: \mathbf{v} \otimes \nabla_{t}\right) \mathrm{d} A \\
& =\omega v_{1}\left(r_{i}\right) \int\left(\mathbf{e}_{\phi} \cdot \mathbf{X} \cdot \mathbf{e}_{r}-\frac{1}{r_{i}} \mathbf{e}_{r} \cdot \mathbf{T}_{i} \cdot \mathbf{e}_{\phi}\right) \mathrm{d} A \equiv \omega^{2} v_{1}\left(r_{i}\right) 2 \pi r_{i} \int_{-\infty}^{0} \tau_{1}(z) \mathrm{d} z
\end{aligned}
$$

At first glance, it may be confusing that the reaction quantity $q_{\phi 1}$ appears in $\mathbf{X}$ as well as in $\mathbf{T}_{i}$. But the two contributions cancel each other and do not influence the power, as is only to be expected. The value of the integral is not finite, but we will only be interested in the apparent shear stress $\tau_{1}(z)$.

We consider the free surface next. Any function $f$ on that surface can be described as follows:

$$
\begin{aligned}
f(r, z=h(r)) & =f(r, z=0)+f,_{z}(r, z=0) h(r)+o(h(r)) \\
& =f(r, z=0)+\omega^{2} f,,_{z}(r, z=0) h_{2}(r)+o\left(\omega^{2}\right)
\end{aligned}
$$

The velocity there must be normal to $\mathbf{n}$ :

$$
\begin{aligned}
0 & =\mathbf{n} \cdot \mathbf{v}=\mathbf{n} \cdot \mathbf{v}_{\mathrm{s}}=\frac{1}{r}\left(\mathbf{n} \times \mathbf{e}_{\phi}\right) \cdot \nabla(r y)(r, z=h(r)) \\
& =-\frac{1}{r}\left(\mathbf{e}_{r}+\omega^{2} h_{2}^{\prime} \mathbf{e}_{z}\right) \cdot \nabla\left(r \omega^{2} y_{2}\right)\left(r, z=\omega^{2} h_{2}(r) 0\right)+o\left(\omega^{2}\right) \\
& =-\omega^{2} \frac{1}{r}\left(r y_{2}\right)_{r}(r, z=0)+o\left(\omega^{2}\right)
\end{aligned}
$$

So $r y_{2}=K=$ const at the surface $z=0$. We may, however, choose $K=0$ because a constant value of $r y_{2}$ gives no contribution to the velocity field. So we arrive at

$$
y_{2}(r, z=0)=0 \quad \Longrightarrow \quad D y_{2}(r, z=0) \equiv\left(y_{2}, r r+\frac{y_{2}, r}{r}-\frac{y_{2}}{r^{2}}+y_{2}, z z\right)(r, z=0) \equiv y_{2}, z z(r, z=0)
$$

and continuity implies $y_{i}=y_{o}=0$, too, as was already mentioned. The dynamic prescriptions are

$$
\mathbf{t}_{e}=\mathbf{0}, \quad \overline{\mathbf{t}}_{e}=\mathbf{0}, \quad \mathbf{T}_{e}=\mathbf{0}
$$

and imply the following boundary conditions

$$
\begin{aligned}
& \mathbf{0}=\overline{\mathbf{t}}_{e}=\mathbf{T}_{3}: \mathbf{n} \otimes \mathbf{n} \\
& \mathbf{0}=\mathbf{t}_{e}=\mathbf{X} \cdot \mathbf{n}-\mathbf{S} \cdot \nabla_{t} \quad \text { with } \quad \mathbf{S}=\mathbf{T}-\mathbf{T}_{e}=\mathbf{T}
\end{aligned}
$$

according to (12) and (17). We need

$$
\mathbf{T}_{3}(r, \phi, z=h(r)) \cdot \mathbf{n}=\left(q_{z 0}\left(\mathbf{1}+\mathbf{e}_{z} \otimes \mathbf{e}_{z}\right)+q_{r 0} \mathbf{e}_{z} \otimes \mathbf{e}_{r}\right)(z=0)
$$




$$
\begin{aligned}
& +\omega\left(q_{\phi 1} \mathbf{e}_{z} \otimes \mathbf{e}_{\phi}+\frac{\alpha_{4}}{4} D v_{1} \mathbf{e}_{z} \otimes \mathbf{e}_{\phi}+\alpha_{5} D v_{1} \mathbf{e}_{\phi} \otimes \mathbf{e}_{z}\right. \\
& +\alpha_{6}\left(v_{1, r z} \mathbf{e}_{\phi} \otimes \mathbf{e}_{r}-\frac{v_{1}, z}{r} \mathbf{e}_{r} \otimes \mathbf{e}_{\phi}+v_{1}, z z \mathbf{e}_{\phi} \otimes \mathbf{e}_{z}\right) \\
& \left.+\frac{\alpha_{7}}{2}\left(v_{1, r z} \mathbf{e}_{r} \otimes \mathbf{e}_{\phi}-\frac{v_{1}, z}{r} \mathbf{e}_{\phi} \otimes \mathbf{e}_{r}+v_{1}, \mathbf{e}_{z} \otimes \mathbf{e}_{\phi}\right)\right)(z=0) \\
& +\omega^{2}\left(\left(q_{z 0, z}\left(\mathbf{1}+\mathbf{e}_{z} \otimes \mathbf{e}_{z}\right)+q_{r 0},_{z} \mathbf{e}_{z} \otimes \mathbf{e}_{r}\right) h_{2}\right. \\
& -\left(q_{r 0}\left(\mathbf{1}+\mathbf{e}_{r} \otimes \mathbf{e}_{r}\right)+q_{z 0} \mathbf{e}_{r} \otimes \mathbf{e}_{z}\right) h_{2}^{\prime} \\
& +\left(q_{z 2}\left(\mathbf{1}+\mathbf{e}_{z} \otimes \mathbf{e}_{z}\right)+q_{r 2} \mathbf{e}_{z} \otimes \mathbf{e}_{r}\right) \\
& -\frac{\alpha_{4}}{4}\left(\left(y_{2, r z z}+\frac{y_{2}, z z}{r}\right)\left(\mathbf{1}+\mathbf{e}_{z} \otimes \mathbf{e}_{z}\right)-D y_{2}, z \mathbf{e}_{z} \otimes \mathbf{e}_{r}\right) \\
& +\alpha_{5}\left(D y_{2}, z \mathbf{e}_{r} \otimes \mathbf{e}_{z}-\left(y_{2}, r z z+\frac{y_{2}, z z}{r}\right) \mathbf{e}_{z} \otimes \mathbf{e}_{z}\right) \\
& +\alpha_{6}\left(y_{2}, r z z \mathbf{e}_{r} \otimes \mathbf{e}_{r}-\left(y_{2}, r z z+\frac{y_{2}, z z}{r}\right) \mathbf{e}_{z} \otimes \mathbf{e}_{z}\right. \\
& +y_{2}, z z z \mathbf{e}_{r} \otimes \mathbf{e}_{z}-\left(D y_{2}, z-y_{2}, z z z \mathbf{e}_{z} \otimes \mathbf{e}_{r}+\frac{y_{2}, z z}{r} \mathbf{e}_{\phi} \otimes \mathbf{e}_{\phi}\right) \\
& +\frac{\alpha_{7}}{2}\left(y_{2}, r z z \mathbf{e}_{r} \otimes \mathbf{e}_{r}-2\left(y_{2}, r z z+\frac{y_{2}, z z}{r}\right) \mathbf{e}_{z} \otimes \mathbf{e}_{z}\right. \\
& -\left(D y_{2}, z-2 y_{2}, z z z\right)\left(\mathbf{e}_{z} \otimes \mathbf{e}_{r}+\mathbf{e}_{r} \otimes \mathbf{e}_{z}\right) \\
& \left.\left.-D y_{2}, z \mathbf{e}_{r} \otimes \mathbf{e}_{z}+\frac{y_{2}, z z}{r} \mathbf{e}_{\phi} \otimes \mathbf{e}_{\phi}\right)\right)(z=0)+o\left(\omega^{2}\right)
\end{aligned}
$$

This gives us the following condition on the free surface

$$
\begin{aligned}
\mathbf{0}= & \mathbf{T}_{3}(r, \phi, z=h(r)): \mathbf{n} \otimes \mathbf{n}=2 q_{z 0}(r, z=0) \mathbf{e}_{z}+\omega\left(\alpha_{5} D v_{1}+\alpha_{6} v_{1}, z z\right)(r, z=0) \mathbf{e}_{\phi} \\
& +\omega^{2}\left(2\left(q_{z 2}+q_{z 0}, h_{2}\right) \mathbf{e}_{z}-2\left(q_{r 0} \mathbf{e}_{z}+q_{z 0} \mathbf{e}_{r}\right) h_{2}^{\prime}+\left(\left(\alpha_{6}+\alpha_{7}\right) y_{2}, z z z+\left(\alpha_{5}-\alpha_{7}\right) D y_{2}, z\right) \mathbf{e}_{r}\right. \\
& \left.-\left(\frac{\alpha_{4}}{2}+\alpha_{5}+\alpha_{6}+\alpha_{7}\right)\left(y_{2}, r z z+\frac{y_{2}, z z}{r}\right) \mathbf{e}_{z}\right)(r, z=0)+o\left(\omega^{2}\right)
\end{aligned}
$$

that can be decomposed into the following statements which will be useful later:

$$
\begin{aligned}
& q_{z 0}(r, z=0)=0 \Longrightarrow q_{z 0, r}(r, z=0)=0 \\
& \left(\alpha_{5} D v_{1}+\alpha_{6} v_{1}, z z\right)(r, z=0)=0 \\
& \left(\left(\alpha_{6}+\alpha_{7}\right) y_{2}, z z z+\left(\alpha_{5}-\alpha_{7}\right) D y_{2}, z\right)(r, z=0)=0 \\
& \left(2\left(q_{z 2}+q_{z 0, z} h_{2}-q_{r 0} h_{2}^{\prime}\right)-\left(\frac{\alpha_{4}}{2}+\alpha_{5}+\alpha_{6}+\alpha_{7}\right)\left(y_{2}, r z z+\frac{y_{2}, z z}{r}\right)\right)(r, z=0)=0 \Longrightarrow \\
& \left(2\left(q_{z 2, r}+q_{z 0}, r z h_{2}+\left(q_{z 0}, z-q_{r 0}, r\right) h_{2}^{\prime}-q_{r 0} h_{2}^{\prime \prime}\right)\right. \\
& \left.\quad-\left(\frac{\alpha_{4}}{2}+\alpha_{5}+\alpha_{6}+\alpha_{7}\right)\left(D y_{2}, z z-y_{2}, z z z z\right)\right)(r, z=0)=0
\end{aligned}
$$

The vectors $\mathbf{e}_{\phi}$ and

$$
\mathbf{e}=\mathbf{e}_{\phi} \times \mathbf{n}=\mathbf{e}_{r}+\omega^{2} h_{2}^{\prime} \mathbf{e}_{z}+o\left(\omega^{2}\right)
$$


constitute an orthonormal basis in the tangential plane. We calculate the surface tensions in cuts normal to each of these vectors, making use of the statements (100) to (104):

$$
\begin{aligned}
& \mathbf{S} \cdot \mathbf{e}=\left(\mathbf{T}_{3} \cdot \mathbf{n}\right) \cdot(\mathbf{1}-\mathbf{n} \otimes \mathbf{n}) \cdot \mathbf{e}=\mathbf{T}_{3}: \mathbf{n} \otimes \mathbf{e} \\
&= q_{r 0}(r, z=0) \mathbf{e}_{z}+\omega\left(\alpha_{6} v_{1}, r z-\frac{\alpha_{7}}{2} \frac{v_{1}, z}{r}\right)(r, z=0) \mathbf{e}_{\phi} \\
&+\omega^{2}\left(q_{r 2} \mathbf{e}_{z}+q_{r 0}, h_{2} h_{2} \mathbf{e}_{z}-q_{r 0} h_{2}^{\prime} \mathbf{e}_{r}+\frac{1}{2}\left(\alpha_{5}+\alpha_{6}+\alpha_{7}\right) \frac{y_{2}, z z}{r} \mathbf{e}_{r}\right. \\
&+\frac{1}{2}\left(\alpha_{5}+3 \alpha_{6}+2 \alpha_{7}\right) y_{2}, r z z \\
& \mathbf{e}_{r} \\
&\left.+\left(\left(\frac{\alpha_{4}}{4}-\alpha_{6}-\frac{\alpha_{7}}{2}\right) D y_{2},,_{z}+\left(\alpha_{6}+\alpha_{7}\right) y_{2},{ }_{z z z}\right) \mathbf{e}_{z}\right)(r, z=0)+o\left(\omega^{2}\right) \\
& \mathbf{S} \cdot \mathbf{e}_{\phi}=\left(\mathbf{T}_{3} \cdot \mathbf{n}\right) \cdot(\mathbf{1}-\mathbf{n} \otimes \mathbf{n}) \cdot \mathbf{e}_{\phi}=\mathbf{T}_{3}: \mathbf{n} \otimes \mathbf{e}_{\phi} \\
&= \omega\left(q_{\phi 1} \mathbf{e}_{z}+\frac{\alpha_{4}}{4} D v_{1} \mathbf{e}_{z}-\alpha_{6} \frac{v_{1}, z}{r} \mathbf{e}_{r}+\frac{\alpha_{7}}{2}\left(v_{1}, r_{z} \mathbf{e}_{r}+v_{1},{ }_{z z} \mathbf{e}_{z}\right)\right)(r, z=0) \\
&+\omega^{2}\left(\frac{1}{2}\left(\alpha_{5}+\alpha_{6}+\alpha_{7}\right) y_{2}, r z z\right. \\
&
\end{aligned}
$$

So the second-order tensor of the surface tension becomes

$$
\mathbf{S}=(\mathbf{S} \cdot \mathbf{e}) \otimes \mathbf{e}+\left(\mathbf{S} \cdot \mathbf{e}_{\phi}\right) \otimes \mathbf{e}_{\phi}
$$

The tangential derivative is

$$
\begin{aligned}
\nabla_{t} & =(\mathbf{1}-\mathbf{n} \otimes \mathbf{n}) \cdot \nabla=\left(\mathbf{e} \otimes \mathbf{e}+\mathbf{e}_{\phi} \otimes \mathbf{e}_{\phi}\right) \cdot\left(\mathbf{e}_{r} \frac{\partial}{\partial r}+\mathbf{e}_{\phi} \frac{\partial}{r \partial \phi}+\mathbf{e}_{z} \frac{\partial}{\partial z}\right) \\
& =\mathbf{e}\left(\frac{\partial}{\partial r}+\omega^{2} h_{2}^{\prime} \frac{\partial}{\partial z}\right)+\mathbf{e}_{\phi} \frac{\partial}{r \partial \phi}
\end{aligned}
$$

The underlined term may be disregarded since we apply $\nabla_{t}$ on $\mathbf{S}$ that only depends on $r$ and $\phi$. So we obtain the following two expressions which are needed in the second boundary condition:

$$
\begin{aligned}
\mathbf{S} \cdot \nabla_{t}= & \frac{\partial(\mathbf{S} \cdot \mathbf{e})}{\partial r}+\frac{(\mathbf{S} \cdot \mathbf{e})}{r} \frac{\partial \mathbf{e}}{\partial \phi} \cdot \mathbf{e}_{\phi}+\frac{\partial\left(\mathbf{S} \cdot \mathbf{e}_{\phi}\right)}{r \partial \phi} \\
= & \left(q_{r 0, r}+\frac{q_{r 0}}{r}\right) \mathbf{e}_{z}(r, z=0) \\
& +\omega \alpha_{6}\left(D v_{1}, z-v_{1}, z z z\right)(r, z=0) \mathbf{e}_{\phi} \\
& +\omega^{2}\left(\left(q_{r 2, r}+\frac{q_{r 2}}{r}\right) \mathbf{e}_{z}+\left(q_{r 0}, r z+\frac{q_{r 0}, z}{r}\right) h_{2} \mathbf{e}_{z}\right. \\
& +\left(-\left(q_{r 0, r}+\frac{q_{r 0}}{r}\right) \mathbf{e}_{r}+q_{r 0, z} \mathbf{e}_{z}\right) h_{2}^{\prime}-q_{r 0} h_{2}^{\prime \prime} \mathbf{e}_{r} \\
& +\frac{1}{2}\left(\alpha_{5}+3 \alpha_{6}+2 \alpha_{7}\right)\left(D y_{2}, z z-y_{2}, z z z z\right) \mathbf{e}_{r}+\left(\alpha_{6}+\alpha_{7}\right)\left(y_{2}, r z z z+\frac{y_{2}, z z z}{r}\right) \mathbf{e}_{z} \\
& \left.+\left(\frac{\alpha_{4}}{4}-\alpha_{6}-\frac{\alpha_{7}}{2}\right)\left(D y_{2}, r z+\frac{D y_{2}, z}{r}\right) \mathbf{e}_{z}\right)(r, z=0)+o\left(\omega^{2}\right)
\end{aligned}
$$

and—with (104)

$$
\begin{aligned}
& \mathbf{X}(r, \phi, z=h(r)) \cdot \mathbf{n}= \\
& -\left(p_{0}+q_{r 0, r}+\frac{q_{r 0}}{r}+2 q_{z 0, z}\right)(r, z=0) \mathbf{e}_{z}+\omega\left(\frac{\alpha_{2}}{2} v_{1, z}-\left(\alpha_{5}+\alpha_{6}\right) D v_{1}, z\right)(r, z=0) \mathbf{e}_{\phi} \\
& +\omega^{2}\left(-\left(p_{2}+q_{r 2, r}+\frac{q_{r 2}}{r}+2 q_{z 2, z}\right) \mathbf{e}_{z}-\left(p_{0}, z+q_{r 0, r z}+\frac{q_{r 0}, z}{r}+2 q_{z 0}, z z\right) h_{2} \mathbf{e}_{z}\right.
\end{aligned}
$$




$$
\begin{aligned}
& +\left(\left(p_{0}+q_{r 0, r}+\frac{q_{r 0}}{r}+2 q_{z 0}, z\right) \mathbf{e}_{r}+q_{r 0}, \mathbf{e}_{z}\right) h_{2}^{\prime}-q_{r 0} h_{2}^{\prime \prime} \mathbf{e}_{r} \\
& -\alpha_{2}\left(\left(y_{2}, r z+\frac{y_{2}, z}{r}\right) \mathbf{e}_{z}-\frac{1}{2} y_{2},{ }_{2 z} \mathbf{e}_{r}\right) \\
& +\left(\frac{1}{2}\left(\frac{\alpha_{4}}{2}+\alpha_{5}+\alpha_{6}+\alpha_{7}\right) y_{2}, z z z z-\frac{1}{2}\left(\alpha_{4}+3 \alpha_{5}+3 \alpha_{6}+2 \alpha_{7}\right) D y_{2}, z z\right. \\
& \left.+\left(\frac{\alpha_{4}}{4}+\frac{\alpha_{7}}{2}\right) D D y_{2}\right) \mathbf{e}_{r} \\
& \left.+\left(\frac{\alpha_{4}}{4}+\alpha_{5}+\alpha_{6}+\frac{\alpha_{7}}{2}\right)\left(D y_{2}, r z+\frac{D y_{2}, z}{r}\right) \mathbf{e}_{z}\right)(r, z=0)+o\left(\omega^{2}\right)
\end{aligned}
$$

When making use of the advanced approach to the reaction stresses, we will restrict our discussion to the case $\beta=0$. Then, Eqs. (52), (53) become linear:

$$
\begin{aligned}
p & =u+t_{2} u^{\bullet} \\
2 \mathbf{q} & =-L^{2}\left(\nabla u+t_{3}\left((\nabla u)^{\bullet}+(\nabla \otimes \mathbf{v}) \cdot \nabla u\right)\right)
\end{aligned}
$$

with

$$
u^{\bullet}=\mathbf{v} \cdot \nabla u, \quad(\nabla u)^{\bullet}=(\mathbf{v} \cdot \nabla) \nabla u
$$

We put

$$
u(r, z)=u_{0}(r, z)+\omega^{2} u_{2}(r, z)+o\left(\omega^{2}\right)
$$

and find $q_{\phi} \equiv 0$ and

$$
\begin{aligned}
p= & u_{0}+\omega^{2}\left(u_{2}+t_{2}\left(y_{2}, z u_{0}, r-\left(y_{2}, r+\frac{y_{2}}{r}\right) u_{0}, z\right)\right)+o\left(\omega^{2}\right) \\
-\frac{2 q_{r}}{L^{2}}= & u_{0}, r+\omega^{2}\left(u_{2, r}+t_{3}\left(y_{2}, z u_{0}, r r+y_{2}, r z u_{0}, r\right.\right. \\
& \left.\left.-\left(y_{2}, r+\frac{y_{2}}{r}\right) u_{0}, r z-\left(D y_{2}-y_{2}, z z\right) u_{0}, z\right)\right)+o\left(\omega^{2}\right) \\
-\frac{2 q_{z}}{L^{2}}= & u_{0},{ }_{z}+\omega^{2}\left(u_{2, z}+t_{3}\left(y_{2}, z u_{0}, r z+y_{2}, z z u_{0}, r\right.\right. \\
& \left.\left.-\left(y_{2}, r+\frac{y_{2}}{r}\right) u_{0}, z z-\left(y_{2} \cdot r z+\frac{y_{2}, z}{r}\right) u_{0}, z\right)\right)+o\left(\omega^{2}\right)
\end{aligned}
$$

The body force per unit mass is

$$
\mathbf{b}=-g \mathbf{e}_{z}
$$

and the acceleration

$$
\mathbf{a}=(\mathbf{v} \cdot \nabla) \mathbf{v}=-\omega^{2} \frac{v_{1}^{2}}{r} \mathbf{e}_{r}+o\left(\omega^{2}\right)
$$

The balance of momentum reads

$$
\begin{aligned}
\mathbf{0}= & \rho(\mathbf{b}-\mathbf{a})+\mathbf{X} \cdot \nabla=\rho(\mathbf{b}-\mathbf{a})+\frac{\alpha_{2}}{2} \Delta \mathbf{v}-\left(\alpha_{5}+\alpha_{6}\right) \Delta \Delta \mathbf{v}-\nabla(p+2 \mathbf{q} \cdot \nabla) \\
= & -\rho g \mathbf{e}_{z}-\nabla\left(p_{0}+2 q_{r 0}, r+2 \frac{q_{r 0}}{r}+2 q_{z 0}, z\right)+\omega\left(\frac{\alpha_{2}}{2} D v_{1}-\left(\alpha_{5}+\alpha_{6}\right) D D v_{1}\right) \mathbf{e}_{\phi} \\
& +\omega^{2}\left(\rho \frac{v_{1}^{2}}{r} \mathbf{e}_{r}+\frac{\alpha_{2}}{2}\left(D y_{2}, z \mathbf{e}_{r}-\left(D y_{2}, r+\frac{D y_{2}}{r}\right) \mathbf{e}_{z}\right)\right. \\
& -\left(\alpha_{5}+\alpha_{6}\right)\left(D D y_{2}, \mathbf{e}_{r}-\left(D D y_{2}, r+\frac{D D y_{2}}{r}\right) \mathbf{e}_{z}\right) \\
& \left.-\nabla\left(p_{2}+2 q_{r 2, r}+2 \frac{q_{r 2}}{r}+2 q_{z 2}, z\right)\right)+o\left(\omega^{2}\right)
\end{aligned}
$$




\section{Evaluation}

Level $\mathbf{0}$ The fluid is at rest. $\omega=0, \mathbf{v}=\mathbf{0}, q_{\phi}=0, h=0$. The boundary condition (97) together with (110), (111) yields

$$
\left(p_{0}+2\left(q_{r 0, r}+\frac{q_{r 0}}{r}+q_{z 0}, z\right)\right)(r, z=0) \mathbf{e}_{z}=\mathbf{0}
$$

so that the integration of the balance of momentum (121) implies

$$
p_{0}+2\left(q_{r 0, r}+\frac{q_{r 0}}{r}+q_{z 0}, z\right)=-\rho g z
$$

The surface tension is according to (108)

$$
\mathbf{S}_{0}=q_{r 0}(r, z=0) \mathbf{e}_{z} \otimes \mathbf{e}_{r}
$$

The boundary condition (89) at the walls gives

$$
q_{r 0}\left(r_{i}, z\right)=q_{r 0}\left(r_{o}, z\right)=0
$$

and imply that $\mathbf{S}_{0}=\mathbf{0}$ at the walls. The solution is governed by the reaction stresses, but the restrictions on them remain poor if we use the classical approach. The advanced approach gives the additional information

$$
p_{0}=u_{0}, \quad q_{r 0}=-\frac{L^{2}}{2} u_{0}, r, \quad q_{z 0}=-\frac{L^{2}}{2} u_{0}, z
$$

If we impose the plausible postulate that $\left|p_{0}, z\right|$ remains finite with $z \rightarrow-\infty$, then we obtain the solution

$$
p_{0}=u_{0}=-\rho g(z-L \exp (z / L)), \quad q_{r 0} \equiv 0, \quad q_{z 0}=\frac{\rho g L^{2}}{2}(1-\exp (z / L)), \quad \mathbf{S}_{0} \equiv \mathbf{0}
$$

These functions give a useful qualitative description of the reaction stresses even if the elastic length $L$ is not known. The stress vectors and tensors acting on the inner wall are according to (83), (84), (85)

$$
\mathbf{X}_{0}\left(r_{i}, \phi, z\right) \cdot \mathbf{e}_{r}=-\left(p_{0}+q_{z 0, z}\right) \mathbf{e}_{r}=\rho g\left(z-\frac{L}{2} \exp (z / L)\right) \mathbf{e}_{r} \equiv \sigma_{0}(z) \mathbf{e}_{r}
$$

and

$$
\mathbf{T}_{i 0}=q_{z 0} \mathbf{e}_{r} \otimes \mathbf{e}_{z}=\frac{\rho g L^{2}}{2}(1-\exp (z / L)) \mathbf{e}_{r} \otimes \mathbf{e}_{z}
$$

If the wall does not consist of a second gradient material and hence cannot accept the last mentioned contribution, then a crust shell will exist and the additional stress vector

$$
-\mathbf{T}_{i 0} \cdot \nabla_{t}=-q_{z 0, z} \mathbf{e}_{r}=\frac{\rho g L}{2} \exp (z / L) \mathbf{e}_{r}
$$

is applied to the wall. Both contributions together yield the classical result

$$
\mathbf{X}_{0} \cdot \mathbf{e}_{r}-\mathbf{T}_{i 0} \cdot \nabla_{t}=\rho g z \mathbf{e}_{r} \equiv \sigma_{0}(z) \mathbf{e}_{r}
$$

The two possibilities are represented in Fig. 1 with the choice $L=r_{o} / 2$.

Level 1 Slow motion of the fluid. Terms with $\omega^{2}$ are neglected. There is no secondary flow, $\mathbf{v}_{S}=\mathbf{0}$. The free surface remains at $z=0$ since $h=0$. The values of $p, q_{r}$ and $q_{z}$ are the same as in the state of rest. We introduce the viscous length

$$
l \equiv \sqrt{\frac{2\left(\alpha_{5}+\alpha_{6}\right)}{\alpha_{2}}}
$$

and apply the abbreviations

$$
v \equiv \frac{\alpha_{4}}{4 \alpha_{6}}, \quad-1 \leq \mu \equiv \frac{\alpha_{7}}{\alpha_{6}} \leq 2, \quad \kappa \equiv \frac{\alpha_{5}}{\alpha_{6}} \geq \frac{\mu}{10}-\frac{2}{5}
$$




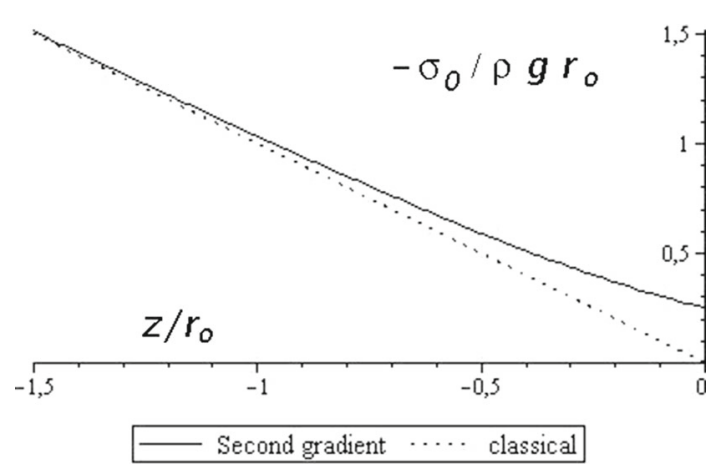

Fig. 1 Pressure at rest (level 0) on the inner wall

The balance of momentum (121) yields the differential equation

$$
\frac{1}{l^{2}} D v_{1}-D D v_{1}=0
$$

The boundary conditions at the walls are according to (86) and (89)

$$
v_{1}\left(r_{k}, z\right)=\frac{\omega_{k}}{\omega} r_{k}, \quad v_{1, r r}\left(r_{k}, z\right)+\frac{\kappa-\mu}{1+\kappa} \frac{1}{r}\left(v_{1, r}\left(r_{k}, z\right)-\frac{\omega_{k}}{\omega}\right)=0 \quad k=i, o
$$

The boundary conditions at the free surface are according to (99) and (97) together with (110), (111)

$$
\begin{aligned}
& \left(v_{1, z z}+\frac{\kappa}{1+\kappa}\left(v_{1, r r}+\frac{v_{1}, r}{r}-\frac{v_{1}}{r^{2}}\right)\right)(z=0)=0 \\
& \left(-v_{1, z}+l^{2} \frac{2+\kappa}{1+\kappa}\left(v_{1, r r z}+\frac{v_{1}, r z}{r}-\frac{v_{1}, z}{r^{2}}\right)+l^{2} v_{1}, z z z\right)(z=0)=0
\end{aligned}
$$

If both cylinders rotate with the same angular velocity $\omega_{i}=\omega_{o}=\omega / \sqrt{2}$, then we have a rigid body rotation of the fluid with

$$
v_{1}=\frac{1}{\sqrt{2}} r, \quad D v_{1}=0, \quad \mathbf{S}_{1}=q_{\phi_{1}}(r) \mathbf{e}_{z} \otimes \mathbf{e}_{\phi}
$$

The classical approach leaves $q_{\phi_{1}}(r)$ undetermined, while the advanced approach gives $q_{\phi_{1}} \equiv 0$ and hence $\mathbf{S}_{1} \equiv \mathbf{0}$.

Of greater interest is the stirring process with $\omega_{i}=\omega, \omega_{o}=0$. A particular solution of the differential equation (134) that does not depend on $z$ is

$$
v_{1 \mathrm{P}}(r)=A r+B \frac{1}{r}+C I_{1}(r / l)+D K_{1}(r / l) \quad \Longrightarrow \quad D v_{1 \mathrm{P}}=\frac{1}{l^{2}}\left(C I_{1}(r / l)+D K_{1}(r / l)\right)
$$

where $I_{1}$ and $K_{1}$ are modified Bessel functions of the first and second kind. The four constants are determined from the boundary conditions (135) but shall not be given here. The boundary condition (137) at $z=0$ is obviously satisfied. The condition (136) is only satisfied in rather special situations: Either $\kappa=0$ or $D v_{1 \mathrm{P}}=0$. The latter possibility implies $C=D=0$ and can only be valid also at the walls if $\mu=-1$ is valid as can be seen from (135). The corresponding solution that does not depend on $z$ is well known from the Navier-Stokes fluid:

$$
v_{1 \mathrm{~N}-\mathrm{St}}=\frac{r_{o} r_{i}^{2}}{r_{o}^{2}-r_{i}^{2}}\left(\frac{r_{o}}{r}-\frac{r}{r_{o}}\right)
$$

In general we must superpose a solution $v_{1 \mathrm{H}}$ of the differential equation that depends on $r$ and $z$ and satisfies the homogeneous boundary conditions. That solution becomes unique if we require $v_{1}, z \rightarrow 0$ with $z \rightarrow-\infty$.

The apparent shear stress $\tau_{1}$ according to (91) becomes

$$
\tau_{1}(z)=\frac{\alpha_{2}}{4 \pi r_{i}}\left(\left(1+\frac{1+\mu}{1+\kappa} \frac{l^{2}}{r_{i}^{2}}\right)\left(v_{1}, r-\frac{v_{1}}{r}\right)-l^{2} D v_{1}, r\right)\left(r=r_{i}, z\right)
$$



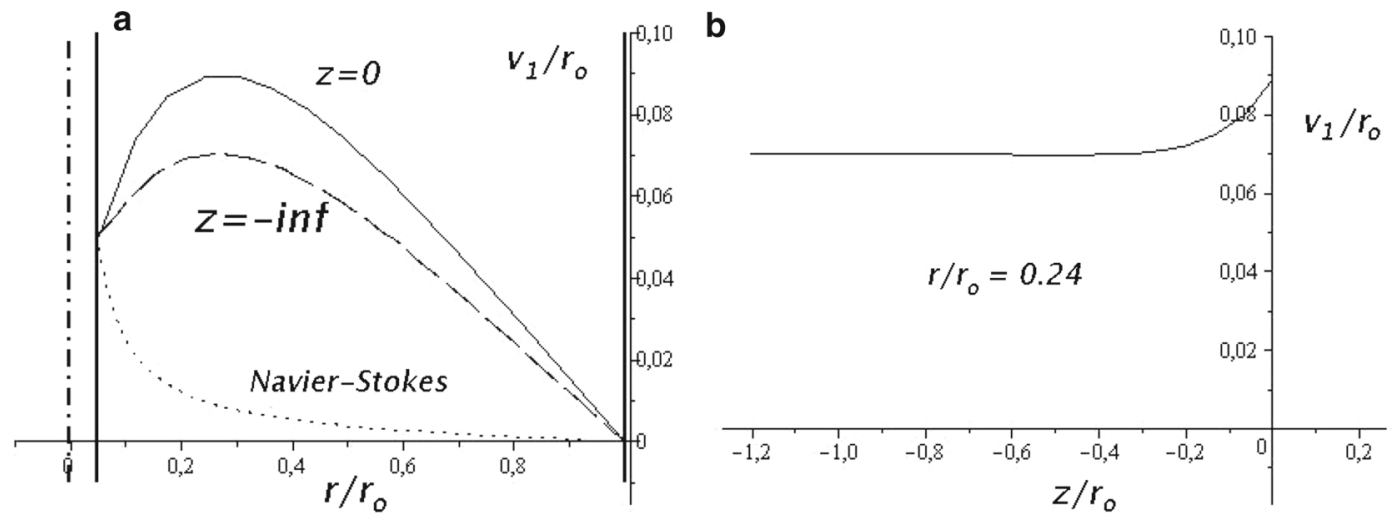

Fig. 2 The velocity profiles of level 1 over $r$ and $z$, respectively

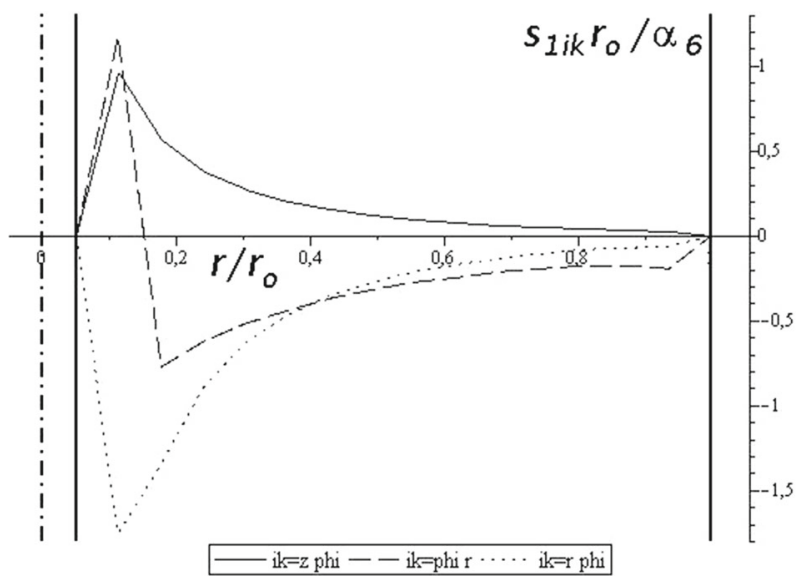

Fig. 3 The components of the surface tension of level 1

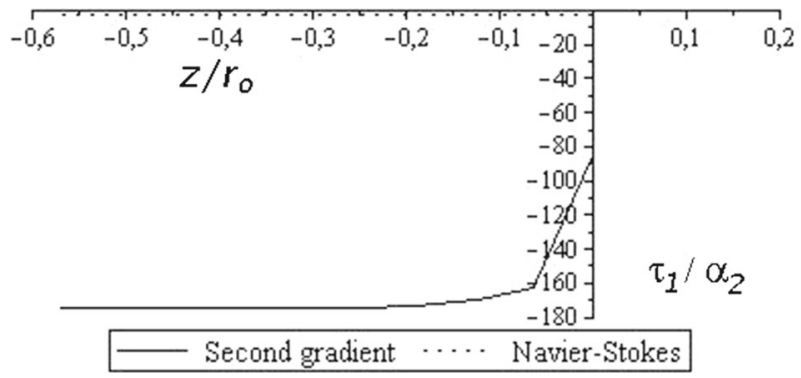

Fig. 4 The apparent shear stress as a measure of the external power over $z$

Level 2 More rapid motion of the fluid. Terms with $\omega^{2}$ are taken into account. The reaction stresses can be eliminated from the balance of momentum (121) by applying the curl operation. The remaining $\phi$-component yields the differential equation

$$
\frac{1}{l^{2}} D D Y-D D D Y=-\frac{1}{r} v_{1} v_{1}, z
$$

with the abbreviation

$$
Y(r, z) \equiv \frac{(1+\kappa) \alpha_{6}}{2 \rho} y_{2}(r, z) \equiv \frac{\alpha_{2} l^{2}}{4 \rho} y_{2}(r, z)
$$

If we treat one of the special cases where $v_{1}$ does not depend on $z$, then $Y \equiv 0$ is a possible solution. So the evolution of a secondary flow is postponed to the level 4 (proportional to $\omega^{4}$ ) as is the case with the Navier- 
Stokes fluid. In general, however, a secondary flow of level 2 (proportional to $\omega^{2}$ ) is necessary to fulfill the balance of momentum.

The boundary conditions at the walls are given by (88) and the $z$-component of (89):

$$
Y\left(r_{k}, z\right)=0, \quad Y, r\left(r_{k}, z\right)=0, \quad r_{k} Y, r r r\left(r_{k}, z\right)+\frac{1+2 \kappa}{1+\kappa} Y, r_{r}\left(r_{k}, z\right)=0 \quad k=i, o
$$

Two boundary conditions on the free surface are obtained from (94) and from (102):

$$
Y(r, z=0)=0, \quad\left(\frac{\kappa-\mu}{1+\kappa}\left(Y, r r z+\frac{Y, r z}{r}-\frac{Y, z}{r^{2}}\right)+Y, z z z\right)(r, z=0)=0
$$

The $r$-component of the boundary condition (97) with (110), (111) together with (122) provides a third boundary condition:

$$
0=\left(\frac{2(1+\kappa)}{l^{2}} Y, z z-2(2 v+2 \kappa+3+2 \mu) D Y, z z+(2 v+2 \kappa+4+3 \mu) Y, z z z z+(2 v+\mu) D D Y\right)(r, z=0)
$$

The $z$-component of the boundary condition (97) at the free surface yields, if (123) is taken into account,

$$
\begin{aligned}
0= & \left(g h_{2}-\frac{1}{\rho}\left(p_{2}+2\left(q_{r 2, r}+\frac{q_{r 2}}{r}+q_{z 2, z}\right)\right)\right. \\
& -\frac{4}{l^{2}}\left(Y, r z+\frac{Y, z}{r}\right)+\frac{2(\kappa+2+\mu)}{1+\kappa}\left(D Y, r z+\frac{D Y, z}{r}\right) \\
& \left.-\frac{2(1+\mu)}{1+\kappa}\left(Y, r z z z+\frac{Y, z z z}{r}\right)\right)(r, z=0)
\end{aligned}
$$

and its derivative with respect to $r$ reads

$$
\begin{aligned}
& 0=\left(g h_{2}^{\prime}(r)-\frac{1}{\rho}\left(p_{2}+2\left(q_{r 2}, r+\frac{q_{r 2}}{r}+q_{z 2, z}\right)\right), r\right. \\
& -\frac{4}{l^{2}}\left(D Y,_{z}-Y, z z z\right)+\frac{2(\kappa+2+\mu)}{1+\kappa}(D D Y, z-D Y, z z z) \\
& \left.-\frac{2(1+\mu)}{1+\kappa}(D Y, z z z-Y, z z z z z)\right)(r, z=0)
\end{aligned}
$$

The $r$-component of the balance of momentum (121) gives

$$
0=\frac{v_{1}^{2}}{r}+\frac{2}{l^{2}} D Y,_{z}-2 D D Y, z-\frac{1}{\rho}\left(p_{2}+2\left(q_{r 2, r}+\frac{q_{r 2}}{r}+q_{z 2, z}\right)\right), r
$$

The last two equations provide information on the shape of the free surface:

$$
\begin{aligned}
g h_{2}^{\prime}(r)= & \left(\frac{v_{1}^{2}}{r}+\frac{2}{l^{2}}\left(3 D Y,_{z}-2 Y, z z z\right)-\frac{2(2 \kappa+3+\mu)}{1+\kappa} D D Y,_{z}+\frac{2(\kappa+2+\mu)}{1+\kappa} D Y, z z z\right. \\
& \left.+\frac{2(1+\mu)}{1+\kappa}\left(D Y,_{z z z}-Y,_{z z z z z}\right)\right)(r, z=0)
\end{aligned}
$$

If there is no secondary flow, then the classical result

$$
h_{2}^{\prime}(r)=\frac{v_{1}(r, z=0)^{2}}{g r}
$$

is valid.

Next we try to find $p_{2}, q_{r 2}$ and $q_{z 2}$ by means of the advanced approach. We go back to (116), (117), (118) and make use of (127):

$$
p_{2}=u_{2}+\frac{2 \rho^{2} g t_{2}}{(1+\kappa) \alpha_{6}}\left(Y, r+\frac{Y}{r}\right)(1-\exp (z / L)
$$




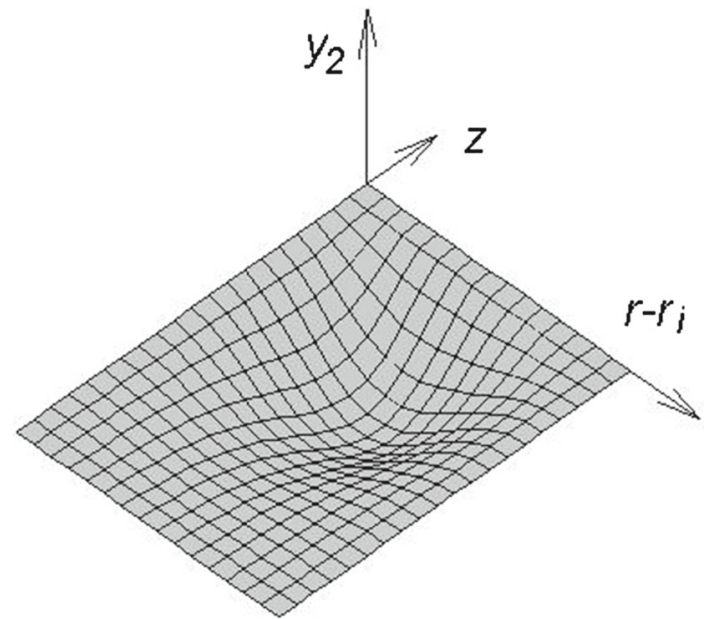

Fig. 5 The function $y_{2}$ that governs the secondary flow, $\min y_{2} \alpha_{6} / \rho r_{o}^{6}=-7 \cdot 10^{-10}$

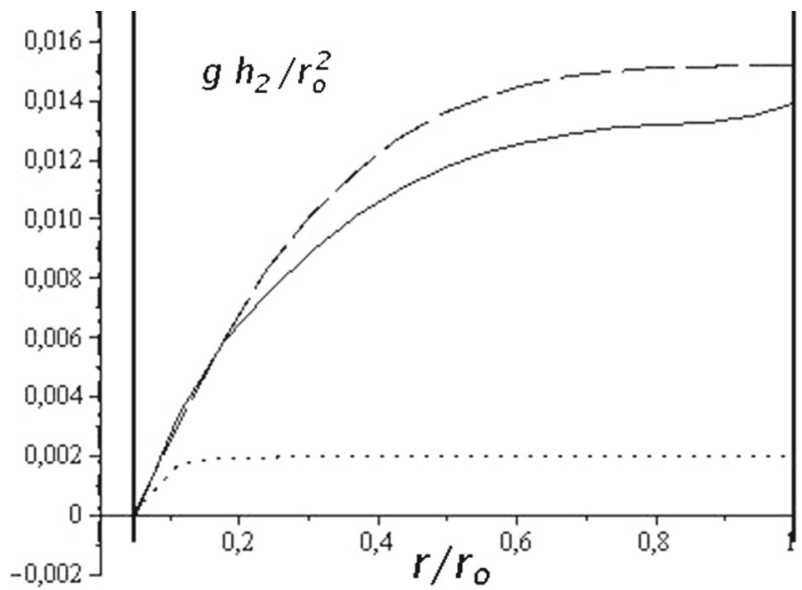

Fig. 6 The shape of the free surface: straight line: actual height, dashed line: classical expression (151) alone, dotted line: Navier-Stokes

$$
\begin{aligned}
-\frac{2 q_{r 2}}{L^{2}} & =u_{2, r}+\frac{2 \rho^{2} g t_{3}}{(1+\kappa) \alpha_{6}}\left(D Y-Y,,_{z z}\right)(1-\exp (z / L)) \\
-\frac{2 q_{z 2}}{L^{2}} & =u_{2}, z+\frac{2 \rho^{2} g t_{3}}{(1+\kappa) \alpha_{6}}\left(\left(Y, r z+\frac{Y, z}{r}\right)(1-\exp (z / L))-\left(Y, r+\frac{Y}{r}\right) \frac{1}{L} \exp (z / L)\right)
\end{aligned}
$$

This implies

$$
\begin{aligned}
p_{2} & +2\left(q_{r 2, r}+\frac{q_{r 2}}{r}+q_{z 2, z}\right) \\
& =u_{2}-L^{2}\left(u_{2, r r}+\frac{u_{2}, r}{r}+u_{2}, z_{z}\right)+\frac{2 \rho^{2} g}{(1+\kappa) \alpha_{6}} t_{3}\left(Y, r+\frac{Y}{r}+2 L\left(Y, r z+\frac{Y, z}{r}\right)\right) \exp (z / L) \\
& +\frac{2 \rho^{2} g}{(1+\kappa) \alpha_{6}}\left(t_{2}\left(Y, r+\frac{Y}{r}\right)-t_{3} L^{2}\left(D Y, r+\frac{D Y}{r}\right)\right)(1-\exp (z / L))
\end{aligned}
$$

The $z$-component of the balance of momentum (121) gives

$$
\frac{1}{\rho}\left(p_{2}+2\left(q_{r 2, r}+\frac{q_{r 2}}{r}+q_{z 2, z}\right)\right),{ }_{z}=-2\left(\frac{1}{l^{2}}\left(D Y,_{r}+\frac{D Y}{r}\right)-D D Y,_{r}-\frac{D D Y}{r}\right)
$$




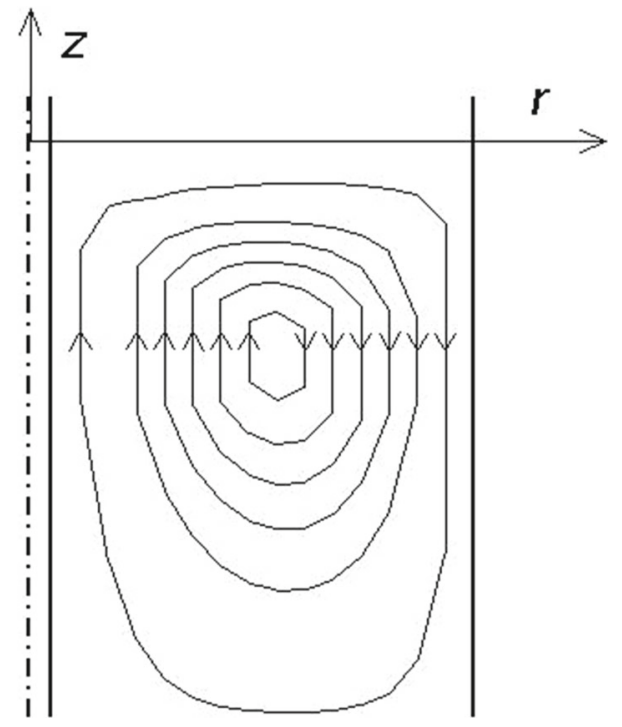

Fig. 7 Stream lines of the secondary flow

We introduce the function

$$
X(r, z) \equiv \int_{\hat{z}=0}^{z} Y(r, \hat{z}) d \hat{z}
$$

and can integrate between 0 and $z$-making use of (147)

$$
\begin{aligned}
\frac{1}{\rho}( & \left.p_{2}+2\left(q_{r 2, r}+\frac{q_{r 2}}{r}+q_{z 2, z}\right)\right)(r, z) \\
= & \left(g h_{2}-\frac{4}{l^{2}}\left(Y, r z+\frac{Y, z}{r}\right)+\frac{2(\kappa+2+\mu)}{1+\kappa}\left(D Y, r z+\frac{D Y, z}{r}\right)\right. \\
& \left.-\frac{2(1+\mu)}{1+\kappa}\left(Y, r z z z+\frac{Y, z z z}{r}\right)\right)(r, z=0) \\
& -2\left(\frac{1}{l^{2}}\left(D X, r+\frac{D X}{r}\right)-D D X, r-\frac{D D X}{r}\right)(r, z) \\
& +2\left(\frac{1}{l^{2}}\left(D X, r+\frac{D X}{r}\right)-D D X, r-\frac{D D X}{r}\right)(r, z=0)
\end{aligned}
$$

Introducing this into (155) we obtain a differential equation for $u_{2}$. Noting that $D Y-Y,_{z z}=Y,_{r r}$ at the walls $r=r_{i}$ and $r=r_{o}$ we obtain boundary conditions from the $r$-component of the condition (89):

$$
u_{2, r}\left(r=r_{k}, z\right)=2 \rho\left(\frac{1}{L^{2}} \frac{1+2 v+\kappa+\mu}{1+\kappa} Y_{, r z}-\frac{\rho g t_{3}}{(1+\kappa) \alpha_{6}} Y_{, r r}(1-\exp (z / L))\right)\left(r=r_{k}, z\right) \quad k=i, o
$$

If we introduce the result (127) into equation (103) and apply (154), we also obtain a boundary condition at the free surface:

$$
u_{2, z}(r, z=0)=-\frac{2}{L^{2}} q_{z 2}(r, z=0)=-\frac{\rho}{L}\left(g h_{2}(r)+\frac{2}{L} \frac{1+2 v+\kappa+\mu}{1+\kappa}\left(Y,_{r z z}+\frac{Y, z z}{r}\right)\right)(r, z=0)
$$

Finally we have to impose the plausible postulate that $u_{2},{ }_{z} \rightarrow 0$ with $z \rightarrow-\infty$. 


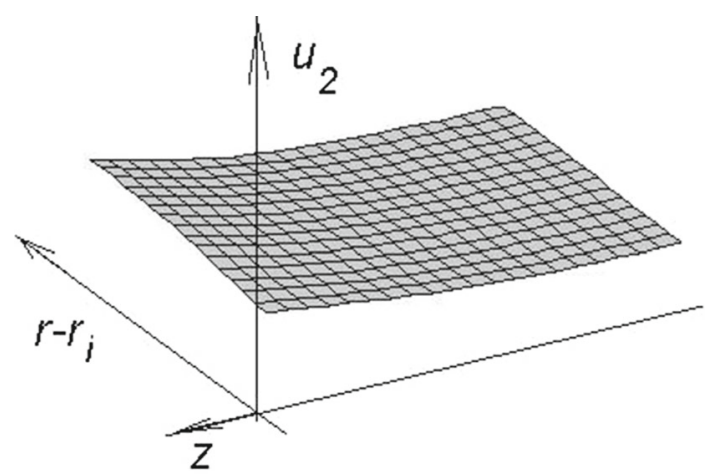

Fig. 8 The function $u_{2}$ that governs the reaction stresses of level 2; the maximum and minimum values of $u_{2} / \rho r_{o}^{2}$ are 0.026 and 0.011 , respectively

\section{Numerical results}

We perform the computation with the geometrical parameter

$$
\frac{r_{o}}{r_{i}}=20
$$

and the material constants

$$
v=0, \quad \kappa=\frac{3}{2}, \quad \mu=\frac{1}{2}, \quad l=L=\frac{r_{o}}{2}, \quad t_{2}=t_{3}=0
$$

While $v_{1 \mathrm{P}}$ was given as an analytic expression, it is hardly possible to construct analytic solutions to the boundary value problems of the functions $v_{1 \mathrm{H}}, Y$ and $u_{2}$. So we apply a difference scheme with a square mesh and a distance between adjacent nodes of $\left(r_{o}-r_{i}\right) / 15$. The function $v_{1 \mathrm{H}}$ is rapidly decreasing so that it is sufficient to evaluate the range $-2\left(r_{o}-r_{i}\right) / 3 \leq z \leq 0$. On the other hand, the evaluation of $Y$ and $u_{2}$ requires the larger range $-4\left(r_{o}-r_{i}\right) / 3 \leq z \leq 0$. We choose as unknowns the values of $v_{1 \mathrm{H}}, Y$ and $u_{2}$ at the nodes, the normal derivatives of $v_{1 \mathrm{H}}$ at $r=r_{i}, r=r_{o}$ and $z=0$ and the normal derivatives of $Y$ and $D Y$ at $z=0$. Figure $2 \mathrm{a}, \mathrm{b}$ gives the velocity profiles of the primary flow at $z=0$ and $z=-\infty$ and the development over $z$, respectively.

The components $s_{1 z \phi}, s_{1 \phi r}$ and $s_{1 r \phi}$ of the corresponding surface tension are given in Fig. 3.

The apparent shear stress $\tau_{1}$ on the inner wall is drawn in Fig. 4 both for the second gradient material and the Navier-Stokes fluid.

An impression of the function $y_{2}(r, z)$ that describes the secondary flow can be found in Fig. 5 .

The shape of the free surface can be seen in Fig. 6.

Figure 7 gives an idea of the stream lines of the secondary flow.

Figure 8 shows the graph of the function $u_{2}(r, z)$.

The surface tension of level 2 is well represented by

$$
\mathbf{S}_{2}=q_{r 2} \mathbf{e}_{z} \otimes \mathbf{e}=-\frac{L^{2}}{2} u_{2, r}(r, z=0) \mathbf{e}_{z} \otimes \mathbf{e}
$$

and is shown in Fig. 9. This is sufficiently precise since the contributions of the derivatives of $y_{2}$ in equations (106), (107) are vanishingly small.

The components of the stress vector of level 2 that act on the inner wall according to (85) are

$$
\begin{aligned}
\sigma_{2 r r}(z) & \equiv \mathbf{e}_{r} \cdot \mathbf{X}_{2}\left(r_{i}, \phi, z\right) \cdot \mathbf{e}_{r} \\
& =\left(-2 \rho \frac{1+v+\kappa+\mu / 2}{1+\kappa} D Y, r z-u_{2}+\frac{L^{2}}{2}\left(2 u_{2}, r r+\frac{u_{2}, r}{r}+u_{2}, z z\right)\right)\left(r_{i}, z\right) \\
\sigma_{2 z r}(z) & \equiv \mathbf{e}_{z} \cdot \mathbf{X}_{2}\left(r_{i}, \phi, z\right) \cdot \mathbf{e}_{r} \\
& =\left(-2 \rho\left(\frac{1}{l^{2}} Y, r r+\frac{1+v+\kappa+\mu / 2}{1+\kappa} Y,{ }_{r r z z}-D D Y\right)+\frac{L^{2}}{2} u_{2}, r z\right)\left(r_{i}, z\right)
\end{aligned}
$$




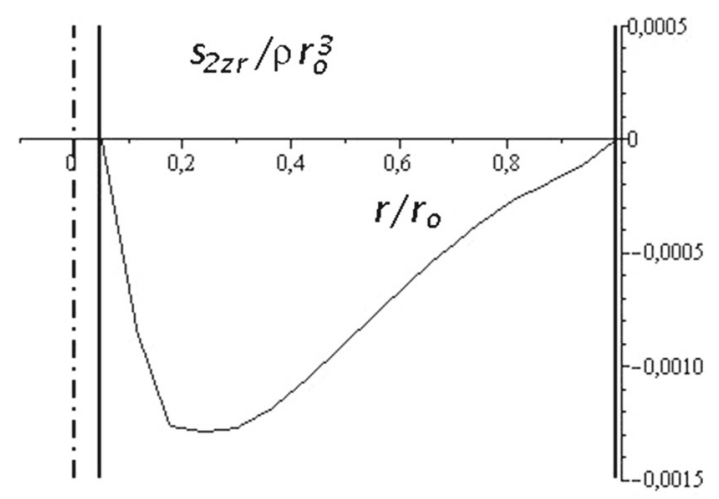

Fig. 9 The surface tension of level 2

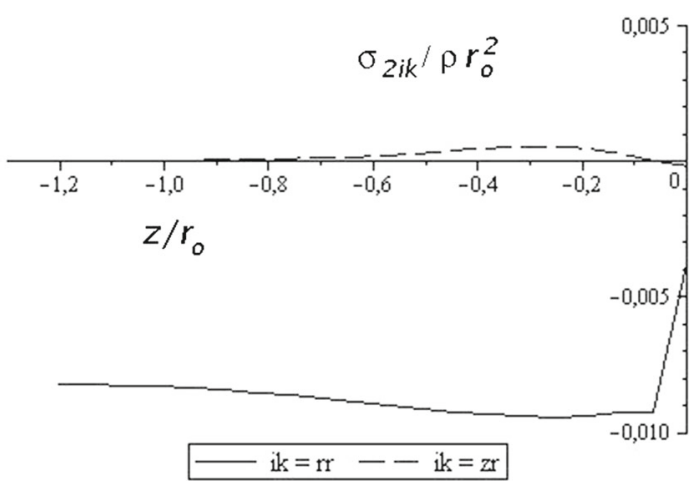

Fig. 10 The stress components of level 2 acting on the inner wall

and are given in Fig. 10. While $\sigma_{2 z r}$ is of local character, the influence of $\sigma_{2 r}$ extends to $z=-\infty$. The tensor $\mathbf{T}_{i 2}$ at the inner wall is not discussed since it is negligibly small everywhere.

The line integral in expression (16) indicates that the surface tension may induce a line force on the boundaries of a free surface. However, equation (127) and Figs. 3 and 9 reveal that these forces of level 0, 1, and 2 are zero both on the boundary $r=r_{i}$ and on the boundary $r=r_{o}$.

\section{Conclusions}

Our investigation brought to light some interesting facts:

Finding 1 It turned out that a free surface of a second gradient material — whatever be its constitutive behavioris endowed with a crust shell that is the basis of a surface tension of dimension force per length.

Finding 2 A scalar field $p$ and a vector field $\mathbf{q}$ appear as reaction quantities with an incompressible second gradient fluid. They are not uniquely determined by the available equations. But this unsatisfactory situation can be defused by an advanced approach: The incompressible material is regarded as the limiting case of a compressible one the stiffness and bulk viscosity of which tend to infinity. Then, $p$ and $\mathbf{q}$ can be derived from one single scalar function.

Finding 3 We presented for the first time the elaboration of the full set of restrictions on the material parameters that ensures nonnegative dissipation in the uncoupled case $\left[\alpha_{8}=\alpha_{9}=0\right.$ in Eqs. (50), (51)].

We discussed a stirring process as an example.

Finding 4 It turned out that not only the primary flow but also a secondary flow must be taken into account in order to determine the shape of the free surface.

Finding $\mathbf{5}$ The reaction fields $p$ and $\mathbf{q}$ remain vague unless the advanced approach is applied. This fact, however, has no influence on the determination of the velocity field. 
Open Access This article is licensed under a Creative Commons Attribution 4.0 International License, which permits use, sharing, adaptation, distribution and reproduction in any medium or format, as long as you give appropriate credit to the original author(s) and the source, provide a link to the Creative Commons licence, and indicate if changes were made. The images or other third party material in this article are included in the article's Creative Commons licence, unless indicated otherwise in a credit line to the material. If material is not included in the article's Creative Commons licence and your intended use is not permitted by statutory regulation or exceeds the permitted use, you will need to obtain permission directly from the copyright holder. To view a copy of this licence, visit http://creativecommons.org/licenses/by/4.0/.

\section{References}

1. de St.-Venant, A.J.C.B.: Rapport sur un mémoire de M. Maurice Lévy, relatif à l'hydrodynamique des liquides homogènes, particulièrement à leur écoulement rectiligne et permanent. Comptes Rendus Acad. Sci. Paris 68, 582-592 (1869)

2. Trostel, R.: Gedanken zur Konstruktion mechanischer Theorien. In: Trostel, R. (ed.) Beiträge zu den Ingenieurwissenschaften, pp. 96-134. Universitätsbibliothek der Technischen Universität, Berlin (1985)

3. Auffray, N., dell'Isola, F., Eremeyev, V.A., Madeo, A., Rosi, G.: Analytical continuum mechanics à la Hamilton-Piola least action principle for second gradient continua and capillary fluids. Math. Mech. Solids 20(4), 375-417 (2015)

4. dell'Isola, F., Gouin, H., Seppecher, P.: Radius and surface tension of microscopic bubbles by second gradient theory. Comptes rendus de l'Académie des Sciences, Série II, Mécanique, physique, chimie, astronomie 320(5), 211-216 (1995)

5. Rosi, G., Giorgio, I., Eremeyev, V.A.: Propagation of linear compression waves through plane interfacial layers and mass adsorption in second gradient fluids. ZAMM J. Appl. Math. Mech. 93(12), 914-927 (2013)

6. Forest, S., Cordero, N.M., Busso, E.P.: First vs. second gradient of strain theory for capillarity effects in an elastic fluid at small length scales. Comput. Mater. Sci. 50(4), 1299-1304 (2011)

7. Silber, G., Trostel, R., Alizadeh, M., Benderoth, G.: A continuum mechanical gradient theory with applications to fluid mechanics. Le Journal de Physique IV, Pr8-365-PR8-373 (1998)

8. Fried, E., Gurtin, M.E.: Tractions, balances, and boundary conditions for nonsimple materials with application to liquid flow at small-length scales. Arch. Ration. Mech. Anal. 182, 513-554 (2006)

9. Bertram, A.: Compendium on Gradient Materials. https://www.lkm.tu-berlin.de/fileadmin/fg49/publikationen/bertram/ Compendium_on_Gradient_Materials_June_2019.pdf (2019)

10. Bertram, A.: On viscous gradient fluids. Contin. Mech. Thermodyn. 32, 1385-1401 (2020)

11. Bertram, A., Forest, S. (eds.): Mechanics of Strain Gradient Materials. Springer, New York (2020)

12. Bertram, A.: An introduction of internal constraints in a natural way. ZAMM 60, T100-T101 (1980)

Publisher's Note Springer Nature remains neutral with regard to jurisdictional claims in published maps and institutional affiliations. 